\title{
On the Effects of Memoryless Nonlinearities on $M$-QAM and DQPSK OFDM Signals
}

\author{
Arsenia Chorti and Mike Brookes, Member, IEEE
}

\begin{abstract}
In the design of RF up-conversion and down-conversion communication links, an issue of special interest is presented by the nonlinear characteristic of analog devices. In this paper, we deal with the effect of memoryless nonlinear distortion on orthogonal frequency-division multiplexing (OFDM) transceivers. We tackle the issue of calculation of the number of intermodulation products with methods from combinatorics theory and derive closed-form expressions for the signal-to-noise ratio (SNR). We deal with third-order nonlinearities alone though the methodology used can be extended to cover higher order nonlinear phenomena. We then proceed to deriving SNR expressions in the presence of a high adjacent channel of the same service and predict the generation of in-band tonal interference. Finally, we generalize to the case of a multichannel OFDM transceiver. In each case, bit-error-rate estimations for differential quadrature phase-shift keying and symbol-error-rate estimations for $M$-quadrature amplitude-modulation constellations are presented and a mapping between circuit characteristics and OFDM performance is outlined.
\end{abstract}

Index Terms-Adjacent channel, bit error rate (BER), combinatorics, digital audio broadcasting (DAB), digital video broadcasting (DVB), discrete multitone (DMT), intermodulation, multicarrier system, nonlinearity, orthogonal frequency-division multiplexing (OFDM), RF circuits, signal-to-noise ratio (SNR), symbol error rate (SER).

\section{INTRODUCTION}

$\mathbf{M}$ ULTICARRIER systems have emerged over recent years as preferred candidates for a variety of wired and wireless applications, allowing higher symbol rates to become attainable. Amongst various implementation approaches, orthogonal frequency-division multiplexing (OFDM) systems [1] stand out owing to their superior performance over frequency-selective channels, their resilience to inter-symbol interference, and their ease in hardware realization by means of fast Fourier transform (FFT). As a result, OFDM has been employed in various applications such as digital video broadcasting (DVB), digital audio broadcasting (DAB), asynchronous digital subscriber line (ADSL) and wireless local area network (LAN).

Nonetheless, because OFDM signals result from the superposition of a high number of modulated carrier signals, they exhibit a high peak-to-mean ratio or crest factor. It is thus likely that some of the RF devices in the up- and down-conversion stages of the communication link will have to cope with high power levels and may

Manuscript received January 6, 2006; revised April 29, 2006. This work was supported by Panasonic System LSI Design Europe.

A. Chorti is with the Electronic Systems Design Group, Department of Electronics and Computer Science, University of Southampton, Southampton SO17 1BJ, U.K. (e-mail: ac2@ecs.soton.ac.uk; ersi.chorti@gmail.com).

M. Brookes is with the Communications and Signal Processing Group, Department of Electrical and Electronic Engineering, Imperial College London, London SW7 2AZ, U.K. (e-mail: mike.brookes@imperial.ac.uk).

Digital Object Identifier 10.1109/TMTT.2006.879129 be driven into the unwanted nonlinear operating region of their input/output characteristic [2]. The effect manifests itself through a severe bit-error-rate (BER) degradation of the OFDM system. Simulation results are presented in [3] and [4].

In [5], Shimbo examined the effects of intermodulation in multicarrier systems. More recently, many authors, [6]-[8] amongst them, have dealt with the impact in OFDM of traveling-wave tube (TWT) [9] or other high power amplifiers (HPAs) that exhibit AM/AM and AM/PM characteristics. The noise due to the nonlinearity was shown in these analyses to be approximately uncorrelated with the OFDM signal that is modeled as a Gaussian random process. However, these results do not apply directly in the case of transistor circuits that are normally modeled as weakly nonlinear memoryless systems [10] because a different nonlinearity model has to be used.

In that context, many authors, amongst them [11] and [12], have tackled the problem by assuming the nonlinearity is memoryless. The OFDM signal is represented as a Gaussian random process and the analysis is based on the derivation of the nonlinearity output autocorrelation function as a function of the autocorrelation of the Gaussian input signal based on statistical signal-processing analyses included in [13]. It is worth noting that Blachman in [14] has given closed-from evaluations of the relevant autocorrelation functions and examined important limiting cases. In order to derive an approximate signal-to-distortion ratio (SDR), van den Bos et al. in [12] assumes that the nonlinear intermodulation noise is dominantly uncorrelated with the OFDM signal. The results presented in this study lend themselves as a useful rough estimation of the system performance degradation.

In our analysis, we intend to lift the restrictions of the approximate analysis in the case of memoryless weakly nonlinear circuits and extract accurate SNR expressions. We represent the OFDM spectrum as a sum of carrier tones missing the central carrier (application in DAB), taking into account their spectral broadening because of phase noise. It is demonstrated that, in the case of weakly nonlinear memoryless systems, phase noise and intermodulation distortion (IMD) can be treated as uncorrelated noise sources, but that their joint effect is more complex than a mere addition of the relevant variances, as presented in [15].

Applying techniques from combinatorics theory, we determine the exact number of IMD products on any given in-band frequency bin and extract closed-form expressions for the carrier signal-to-noise ratio (SNR) and OFDM symbol-error-rate (SER) or BER according to the modulation scheme. Our approach offers an insight into the way the IMD products are generated and into the degree of phase correlation between the OFDM signal and the IMD. To demonstrate the benefit from following a more accurate analysis, we compare our results with the results of [12]. 
The use of combinatorics was introduced quite early and, in [16], Wescott discusses the case of multicarrier FM signals through TWT amplifiers. Pedro and de Carvalho in [17] have calculated many important IMD figures-of-merit in regard to third-order nonlinear circuits. In [18], Liu presents an approximate SNR evaluation. In [19], Boulejfen et al. applied the methodology in multitone signals through fifth-order nonlinear distortion and derived new closed-form expressions for the relevant figures-of-merit. In this study, we are mainly interested in deriving closed-form expressions for the carrier SNR to allow for using the results for BER and SER estimations.

Calculations of the number of IMD products due to thirdorder nonlinearities already exist in the literature (e.g., [16] and [19]). Extending the previous use of combinatorics, we examine the effect of IMD distortion in the presence of phase noise and of additive Gaussian noise channel. Subsequently, we present an analysis for the case of a multitone channel received along with a high adjacent channel at any spacing and the generalization to multichannel multitone excitation. Although our derivations follow the DAB specifications (suppressed central carrier), the results practically apply in the general case as well. It is worth noting that the proposed combinatorics approach leads to much simpler SNR expressions if the OFDM envelope is continuous.

This paper is organized as follows. In Section II, we present the nonlinearity model we will use in our analysis and discuss the suitability of OFDM signals for the methodology derived from combinatorics. In Section III, we deal with a single OFDM channel through a third-order nonlinearity, including band-limited flat spectral density noise and common to all carriers phase noise. A comparison between our results and the results assuming uncorrelated IMD distortion [12] is included. In Section IV, we consider the case of reception of an OFDM channel along with a high power adjacent channel of the same service and discuss issues concerning the reduction of the receiver effective dynamic range. In Section V, we generalize for the case of multiple adjacent channels and highlight the restrictions imposed on the RF circuit designer by a given OFDM service layout. Conclusions are presented in Section VI.

\section{WEAKLY NONLINEAR CIRCUITS AND THE USE OF COMBINATORICS}

According to the theory of weakly nonlinear systems, we can represent a component's input/output characteristic by a truncated power series around the dc operating point as long as a frequency-independent model is appropriate [20]. On the other hand, if the component's nonlinearities are not memoryless, Volterra analysis should be used instead for the representation of the component's characteristic [21].

In the following, we will assume that: 1) the circuit is weakly nonlinear and 2) the circuit input/output characteristic $v_{\text {out }}(t)=$ $f\left[v_{\text {in }}(t)\right]$ can be approximated by the first four terms of its Taylor series expansion

$$
\begin{array}{r}
f\left[v_{\text {in }}(t)\right] \simeq \sum_{n=0}^{3} a_{n} v_{\text {in }}^{n}=a_{0}+a_{1} v_{\text {in }}(t)+a_{2} v_{\text {in }}^{2}(t)+a_{3} v_{\text {in }}^{3}(t), \\
\text { where } a_{n}=\left.\frac{1}{n !} \frac{d^{n} f\left(v_{\text {in }}\right)}{d v_{\text {in }}^{n}}\right|_{v_{\text {in }}=0} .
\end{array}
$$

The above approximation is realistic for a wide range of lownoise amplifiers, mixers, and baseband amplifiers [22], [23].

The use of combinatorics and of the generating function technique in particular [24], [25] to calculate the number of IMD products presents, in the general case, clear limitations. The problem to be solved is particularly complex if the multitone excitation of the nonlinearity have an irregular frequency spacing or if the tones are not of the same amplitude. However, in OFDM systems, we do indeed have a regular carrier spacing due to the inverse fast Fourier transform (IFFT) in the transmitter.

Furthermore, since we are interested in SNR expressions, the requirement of carriers of the same amplitude can actually be reduced to the following. The random variables (RVs) representing the carrier amplitudes should be independent or at least uncorrelated up to the sixth order and the modulation phases need to be independent RVs with known moments up to the sixth order. The above requirements are reasonable approximations in many cases of real broadcasting systems and are presumed valid in the rest of this paper. The combinatorial approach is well suited to DAB as the carriers are designed to have the same amplitude. The effect of frequency-selective broadcasting channels is not examined in this study.

The main difficulty in using combinatorics for IMD distortion analysis is in the evaluation of the coefficients of products of infinite-length polynomials in a parametric closed form. In the remainder of this paper, these coefficients have been readily calculated for all the examined cases.

\section{OFDM SignalS THROUGH THIRD-ORDER NONLINEARITY}

In Section III-A, we calculate the number of in-band IMD products in the case of a third-order nonlinear circuit with OFDM input degraded with common to all carriers phase noise. In Section III-B, we derive the SNR expression including the effect of phase noise, while in Section III-C, we also account for the presence of band-limited white-like noise. Finally, in Section III-D, we present the mapping of the circuit nonlinearities to BER and SER estimations.

\section{A. OFDM With Phase Noise Through Third-Order Nonlinearity}

To begin our analysis with, we model the nonlinear RF component characteristic as a third-order polynomial. Modeling the circuit nonlinearity with such a low-order polynomial is actually an efficient approximation in many real systems since the third-order nonlinearity is responsible for most of the IMD distortion [16]. Furthermore, we neglect even-order nonlinearities because the resulting IMD products will be out of the OFDM band. Without loss of generality, we may neglect the dc term $a_{0}$ and express the output of the nonlinear circuit as

$$
v_{\text {out }}(t)=a_{1} v_{\text {in }}(t)+a_{3} v_{\text {in }}^{3}(t)
$$

where $a_{1}$ and $a_{3}$ are the linear gain and third-order nonlinearity coefficient, respectively, while $v_{\text {in }}$ is the input OFDM voltage. Commonly, for the assessment of a circuit's nonlinear characteristics, circuit designers make use of the 1-dB compression point or second- and third-order intercept points. In the following, we will characterize the nonlinearity through the output-inferred 
third-order intercept point (OIP3) [26]. Its dependence on the linear gain is cubic, while it is inversely proportional to $a_{3}$ :

$$
\text { OIP3 }=\left|\frac{2}{3} \frac{a_{1}^{3}}{a_{3}}\right|
$$

The analysis can be summarized as follows. If we represent the OFDM signal as a sum of carrier tones, then the third-order term resulting from substitution in (1) is expressed as two triple sums; one centered in the OFDM band and the second at thrice the frequency. Neglecting out-of-band terms, the evaluation of the output SNR requires the determination of the number of in-band IMD components and of their phase relation to the given carrier. For the evaluation of the number of IMD terms, we can either perform a threefold convolution of the sum of the Dirac deltas representing the OFDM spectrum or use counting methods from combinatorics theory. Subsequently, further use of combinatorics enables us to count the number of IMD products that are in-phase with a given OFDM carrier and, hence, add with it in amplitude. The effect of the remaining IMD components depends on the statistics of the modulation phases. As long as the RVs that represent the OFDM carrier phases can be assumed independent and the IMD products have null averages, the problem is simplified and the out-of-phase IMD tones will add in power with each other and with the OFDM carrier.

We start the analysis by assuming that $v_{\text {in }}$ represents an OFDM signal corrupted only by phase noise (common to all carriers $^{1}$ )

$$
v_{\text {in }}(t)=\sum_{\substack{k=0 \\ k \neq \frac{N}{2}}}^{N} \Re\left(z_{k} e^{j\left[\left(\omega_{d}+k \omega_{u}\right) t+\theta_{k}+\phi\right]}\right) .
$$

The random process $\phi$ accounts for the OFDM signal phase noise from oscillators in earlier stages and $\Re(\cdot)$ denotes the real value. The lower frequency of the OFDM channel is $\omega_{d}$, the carrier spacing is $\omega_{u}$, and $z_{k}$ and $\theta_{k}$ are the $k$ th carrier amplitude and phase. We assume that the RVs $\theta_{k}$ are independent and that the process $e^{j \theta_{k}}$ is zero mean, which is the case for $M$ quadrature amplitude modulation ( $M$-QAM). For differential quadrature phase-shift keying (DQPSK), to ensure that the above is valid, we need to further assume that the transmitted symbols are uncorrelated. Finally, we consider OFDM signals missing the central carrier and of an even number of carriers $N$ (application in $\mathrm{DAB}$ ).

The output voltage can be expressed as in (4), obtained by substitution of (3) into (1) as follows:

$$
\begin{aligned}
& v_{\text {out }}(t) \\
& =a_{1} \sum_{k} \Re\left(z_{k} e^{j\left[\left(\omega_{d}+k \omega_{u}\right) t+\theta_{k}+\phi\right]}\right) \\
& \quad+\frac{a_{3}}{4} \sum_{k, l, m} \Re\left(z_{k} z_{l} z_{m} e^{j\left[\left(3 \omega_{d}+(k+l+m) \omega_{u}\right) t+\left(\theta_{k}+\theta_{l}+\theta_{m}\right)+3 \phi\right]}\right) \\
& \quad+\frac{3 a_{3}}{4} \sum_{k, l, m} \Re\left(z_{k} z_{l} z_{m} e^{j\left[\left(\omega_{d}+(k+l-m) \omega_{u}\right) t+\left(\theta_{k}+\theta_{l}-\theta_{m}\right)+\phi\right]}\right) .
\end{aligned}
$$

${ }^{1}$ Phase noise generated by mixing with a noisy oscillator is common to all carriers.
TABLE I

MOMENTS OF $M$-QAM AMPLITUDES

\begin{tabular}{ccc}
\hline$M$ & $\left\langle z^{4}\right\rangle$ & $\left\langle z^{6}\right\rangle$ \\
\hline \hline 4 & $\left\langle z^{2}\right\rangle^{2}$ & $\left\langle z^{2}\right\rangle^{3}$ \\
8 & $\frac{13}{9}\left\langle z^{2}\right\rangle^{2}$ & $\frac{7}{3}\left\langle z^{2}\right\rangle^{3}$ \\
16 & $\frac{33}{25}\left\langle z^{2}\right\rangle^{2}$ & $\frac{49}{25}\left\langle z^{2}\right\rangle^{3}$ \\
32 & $\frac{109}{81}\left\langle z^{2}\right\rangle^{2}$ & $\frac{503}{242}\left\langle z^{2}\right\rangle^{3}$ \\
64 & $\frac{29}{21}\left\langle z^{2}\right\rangle^{2}$ & $\frac{6871}{3087}\left\langle z^{2}\right\rangle^{3}$ \\
\hline
\end{tabular}

Equation (4) highlights two important conclusions. Firstly, the phase noise is not altered by the nonlinearity for in-band products [the third term in (4)] while it is tripled in the out-ofband products [the second term in (4)]. Secondly, we need to evaluate up to the sixth-order moments of the modulation amplitudes RVs for the calculation of the nonlinearity output power. If the modulation scheme is either $M$-QAM or DQPSK, then the amplitudes are generated by the general expression [27]

$$
\begin{array}{r}
z=\sqrt{\left(2 \zeta-1-M_{1}\right)^{2}+\left(2 \eta-1-M_{2}\right)^{2}} d, \\
\quad \text { where, } \zeta=1, \ldots, M_{1}, \eta=1, \ldots, M_{2} .
\end{array}
$$

The RVs $\zeta$ and $\eta$ are independent and the number of constellation points equals $M=M_{1} M_{2}$. We only need to calculate second-, fourth-, and sixth-order moments. In Table I, we have evaluated for various $M$-QAM constellations the fourth- and sixth-order moments as a function of the second-order moment $\left\langle z^{2}\right\rangle$. The case of DQPSK is covered by 4-QAM. We also assume the use of a rectangular time window, which is typical in OFDM systems.

As shown in (4), the in-band intermodulation products are at frequencies $\omega_{d}+(k+l-m) \omega_{u}$. Therefore, to calculate their number, we have to compute the number of possible ways for an IMD product to appear at a frequency

$$
\omega_{b}=\omega_{d}+(k+l-m) \omega_{u}=\omega_{d}+b \omega_{u}
$$

in the bandpass $\omega_{b} \in\left[\omega_{d}, \omega_{d}+N \omega_{u}\right]$. This problem is equivalent to computing the number of integer solutions of the equation

$$
\begin{aligned}
k+l-m & =b \text { with conditions } \\
0 & \leq k, l, m \leq N \quad k, l, m \neq 0.5 N \quad 0 \leq b \leq N .
\end{aligned}
$$

We denote the set of the possible combinations on any given frequency $\omega_{b}$ as $U(b, N)$. Making use of combinatorics theory [24], [25], we can identify the size $|U|$ of $U(b, N)$ from the generating function $G(x)$ of (5)

$$
\begin{aligned}
G(x)= & \left(1+x+x^{2}+\ldots+x^{N}-x^{\frac{N}{2}}\right)^{2} \\
& \times\left(1+x^{-1}+x^{-2}+\ldots+x^{-N}-x^{-\frac{N}{2}}\right) \\
= & x^{-N}\left(\frac{1-x^{N+1}}{1-x}-x^{\frac{N}{2}}\right)^{3} .
\end{aligned}
$$

The number $|U|$ of in-band IMD products is derived in Appendix I. We distinguish four subsets of $U$ according to 
TABLE II

IMD SUBSETS

\begin{tabular}{cccc}
\hline Subset & $\omega_{b}$ & Ampl. & $\theta_{b}$ \\
\hline \hline$T_{1}$ & $\omega_{b}+\omega_{b}-\omega_{b}$ & $0.75 z_{b}^{3}$ & $\theta_{b}$ \\
$T_{2}$ & $\omega_{k}+\omega_{k}-\omega_{l}$ & $0.75 z_{k}^{2} z_{l}$ & $2 \theta_{k}-\theta_{l}$ \\
$T_{3}$ & $\omega_{b}+\omega_{l}-\omega_{l}$ & $1.5 z_{b} z_{l}^{2}$ & $\theta_{b}$ \\
$T_{4}$ & $\omega_{k}+\omega_{l}-\omega_{m}$ & $1.5 z_{k} z_{l} z_{m}$ & $\theta_{k}+\theta_{l}-\theta_{m}$ \\
\hline
\end{tabular}

the phase relation of the above IMD products in regard to the carrier $\Re\left(z_{b} e^{j\left[\left(\omega_{d}+b \omega_{u}\right) t+\theta_{b}\right]}\right)$, as described in Table II.

In the subsets $T_{3}$ and $T_{4}$, two terms arise with the same frequency and phase by interchanging $\omega_{b}$ and $\omega_{l}$ or $\omega_{k}$ and $\omega_{l}$, respectively, resulting in a term with double the amplitude. The sizes $\left|T_{1}\right|,\left|T_{2}\right|,\left|T_{3}\right|$, and $\left|T_{4}\right|$ of the respective subsets are included in Appendix I.

We note that $|U|$ is of the order $\left(N^{2}\right),\left|T_{1}\right|$ is the unit, $\left|T_{2}\right|$ and $\left|T_{3}\right|$ are of the order $(N)$, and $\left|T_{4}\right|$ is of the order $\left(N^{2}\right)$. In the case of DAB where $N=1536$, it follows that the number of IMD products is 1767167 on the carrier next to the central frequency, of which, 1536 are in-phase with it.

\section{B. Derivation of the SNR at the Output of the Nonlinearity}

We now have to identify how the various subsets of IMD products affect the useful signal. We begin by noticing that subsets $T_{1}$ and $T_{3}$ are in-phase with the carrier at $\omega_{b}$ and will, therefore, add with it in amplitude. According to the sign of the third-order nonlinearity coefficient $a_{3}$, those two subsets will provide either gain enhancement or gain compression to the wanted signal, evaluated as

$$
\Delta a_{1}=\left\langle a_{1}-\frac{3}{4} a_{3} z_{b}^{2}\left|T_{1}\right|+\frac{3}{2} a_{3} \sum_{i=1}^{\left|T_{3}\right|} z_{l, i}^{2}\right\rangle .
$$

It follows that the gain enhancement/compression expressed in (8) depends on $a_{1}, a_{3}, N, M$, and $b$. It increases with decreasing $M$ and increasing $N$.

The useful output power $P_{c}(b, N)$ on a carrier at a frequency bin $\omega_{b}$ is

$$
\begin{aligned}
P_{c}(b, N)= & \frac{1}{2}\left\langle\left[a_{1} z_{b}+\frac{3}{4} a_{3} z_{b}^{3}\left|T_{1}\right|+\frac{3}{2} a_{3} z_{b} \sum_{i=1}^{\left|T_{3}\right|} z_{l, i}^{2}\right]^{2}\right\rangle \\
=\frac{1}{2}[ & a_{1}^{2}\left\langle z^{2}\right\rangle+\frac{9}{16} a_{3}^{2}\left\langle z^{6}\right\rangle+\frac{9}{4} a_{3}^{2}\left|T_{3}\right|\left\langle z^{2}\right\rangle\left\langle z^{4}\right\rangle \\
& +\frac{9}{2} a_{3}^{2}\left(\begin{array}{c}
\left|T_{3}\right| \\
2
\end{array}\right)\left\langle z^{2}\right\rangle^{3}+\frac{3}{2} a_{1} a_{3}\left\langle z^{4}\right\rangle \\
& \left.+\frac{9}{4} a_{3}^{2}\left|T_{3}\right|\left\langle z^{2}\right\rangle\left\langle z^{4}\right\rangle+3 a_{1} a_{3}\left|T_{3}\right|\left\langle z^{2}\right\rangle^{2}\right]
\end{aligned}
$$

where $\left(\begin{array}{l}x \\ y\end{array}\right)=x ! / y !(x-y)$ ! denotes the binomial coefficient.

On the other hand, subsets $T_{2}$ and $T_{4}$ correspond to intermodulation noise. In order to derive the intermodulation noise power, we need to examine whether the IMD products included in subsets $T_{2}$ and $T_{4}$ will add in amplitude or in power. As long as the modulation phases' probability density function is symmetrical in regard to both axes of the constellation diagram, IMD terms in $T_{2}$ and $T_{4}$ are mutually uncorrelated. The

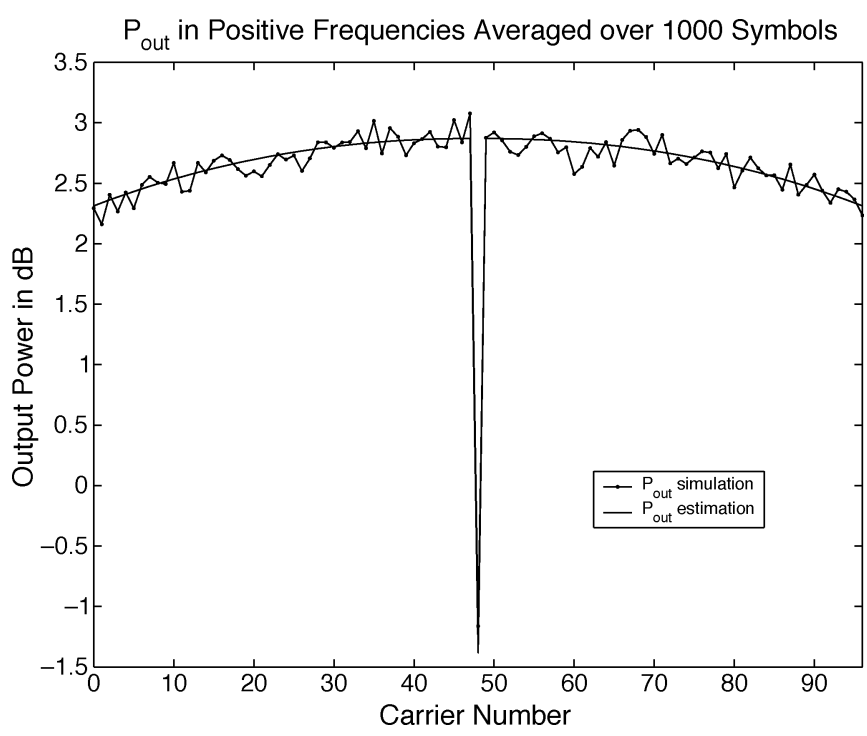

Fig. 1. Output power as estimated analytically and through simulation.

proof can be found in Appendix IV. This criteria is fulfilled for $M$-QAM and DQPSK so that the intermodulation noise $P_{\mathrm{IM}}(b, N)$ can be expressed as

$$
\begin{aligned}
P_{\mathrm{IM}}(b, N) & =\frac{1}{2}\left\langle\left[\frac{3}{4} a_{3} z_{k}^{2} \sum_{i=1}^{\left|T_{2}\right|} z_{l, i}\right]^{2}+\left[\frac{6}{4} a_{3} z_{m} z_{n} \sum_{i=1}^{\left|T_{4}\right|} z_{p, i}\right]^{2}\right\rangle \\
& =\frac{9}{32} a_{3}^{2}\left(\left|T_{2}\right|\left\langle z^{4}\right\rangle\left\langle z^{2}\right\rangle+4\left|T_{4}\right|\left\langle z^{2}\right\rangle^{3}\right) .
\end{aligned}
$$

In Fig. 1, we plot the estimated overall output power $P_{\text {out }}=$ $P_{c}+P_{\mathrm{IM}}$ along with simulation results in the absence of phase noise $\phi=0$. The simulated OFDM output power was evaluated by realizing threefold convolution of QPSK OFDM with $N=96$ carriers and was averaged over 1000 OFDM symbols. The third-order nonlinearity was chosen such that OIP3 $=$ $18.24 \mathrm{dBm}$, while $a_{1}=1$ and $\left\langle z^{2}\right\rangle=2 / N$. The good agreement of the results validates our output power estimator $P_{\text {out }}$.

In the absence of phase noise, the orthogonality of the carriers is not affected by the nonlinearity and we can use (9) and (10) for the SNR evaluation at the output of the FFT. However, in the presence of phase noise, we need to further account for energy spillage between consecutive carriers.

Assuming phase noise is small, it is shown in [28] that the effect of phase noise on OFDM can be decomposed into two terms, i.e., the common phase error (CPE) and the inter-carrier interference (ICI). Their variances $\sigma_{0}^{2}$ and $\sigma_{m}^{2}$, respectively, as a function of the phase noise spectrum $|\Phi(f)|^{2}$, are given as follows:

$$
\begin{aligned}
\sigma_{0}^{2} & =\int_{-\infty}^{\infty} \operatorname{sinc}^{2}\left(\frac{f}{f_{u}}\right)|\Phi(f)|^{2} d f \\
\sigma_{m}^{2} & =\int_{-\infty}^{\infty} \operatorname{sinc}^{2}\left(\frac{f}{f_{u}}\right)\left|\Phi\left(f-m f_{u}\right)\right|^{2} d f
\end{aligned}
$$

where $f_{u}=\omega_{u} / 2 \pi$.

The CPE demonstrates the effect of the broadening of the carrier spectrum, while the ICI results from the effect of energy 
spillage from neighboring carriers. Therefore, IMD terms on the same frequency as the carrier will contribute in terms of CPE, while IMD terms on the remaining carriers will contribute in terms of ICI. As a result, the SNR of the carrier at frequency $\omega_{b}$, due to both phase noise and third-order nonlinearity, can be expressed as

$$
\mathrm{SNR}=\frac{P_{c}(b, N)}{P_{\mathrm{IM}}(b, N)+\sigma_{\mathrm{CPE}}^{2}(b, N)+\sigma_{\mathrm{ICI}}^{2}(b, N)}
$$

where

$$
\begin{aligned}
\sigma_{\mathrm{CPE}}^{2}(b, N) & =\left(P_{c}(b, N)+P_{\mathrm{IM}}(b, N)\right) \sigma_{0}^{2} \\
\sigma_{\mathrm{ICI}}^{2}(b, N) & =\sum_{m \neq b}\left(P_{c}(b-m, N)+P_{\mathrm{IM}}(b-m, N)\right) \sigma_{m}^{2} .
\end{aligned}
$$

The CPE is not Gaussian and, therefore, the SNR expression (13) cannot be directly used for SER or BER estimation.

An important conclusion of this analysis is that the CPE is not "common" to all carriers in the presence of nonlinearities. Unlike in a previous study [15], we have readily shown that the amount of CPE depends on the carrier index $b$ and on the number of OFDM carriers $N$. This effect should be taken into consideration in compensation strategies.

\section{OFDM and Noise Through Third-Order Nonlinearity}

We now proceed by assuming that the input signal is expressed as $v_{\text {in }}(t)+n(t)$, where $v_{\text {in }}(t)$ is given in (3) and $n(t)$ is band-limited additive noise of flat power spectral density $N_{0}$. The OFDM band is included in the band-limited white-like noise band. We note that

$$
\begin{aligned}
v_{\text {out }} & =a_{1}\left(v_{\text {in }}+n\right)+a_{3}\left(v_{\text {in }}+n\right)^{3} \\
& =\underbrace{a_{1} v_{\text {in }}}_{C}+\underbrace{a_{3} v_{\text {in }}^{3}}_{U}+\underbrace{a_{1} n+a_{3}\left(3 v_{\text {in }}^{2} n+3 v_{\text {in }} n^{2}+n^{3}\right)}_{V} .
\end{aligned}
$$

The terms in set $C$ represent the amplified OFDM signal, while set $U$ contains the third-order IMD products previously derived. Set $V$ contains the terms resulting from the intermodulation of the additive white noise with the OFDM channel.

To account for the noise power of terms in $V$, we identify $a_{1} n(t)+3 a_{3} v_{\text {in }}^{2}(t) n(t)$ as the dominant term within this set. We assume that the bandwidth of $n(t)$, on either side of the central OFDM frequency, is at least equal to the OFDM bandwidth, so as to ensure that the spectrum of $3 a_{3} v_{\mathrm{in}}^{2}(t) n(t)$ is flat in the OFDM band. In that case, exploiting the independence of the processes $v_{\text {in }}^{2}(t)$ and $n(t)$, we can calculate the resulting noise power $P_{0}$ per carrier as the product of the variances of the respective noise terms. Assuming a rectangular time window is employed at the OFDM receiver, the variance of $n(t)$ in a carrier spacing bandwidth is given by

$$
\sigma_{n, c}^{2}=\frac{N_{0}}{2 \pi} \int_{-\infty}^{\infty} \operatorname{sinc}^{2}\left(\frac{\omega}{\omega_{u}}\right) d \omega=N_{0} \frac{\omega_{u}}{2 \pi} .
$$

Therefore, the noise power per carrier due to the terms $a_{1} n(t)+$ $3 a_{3} v_{\text {in }}^{2}(t) n(t)$ is evaluated as

$$
P_{0}=a_{1} N_{0} \frac{\omega_{u}}{2 \pi}+9 a_{3}^{2} N_{0} \frac{\omega_{u}}{2 \pi} \sigma_{v_{\mathrm{in}}^{2}}^{2}
$$

where $\sigma_{v_{\mathrm{in}}^{2}}^{2}$ is the variance of $v_{\mathrm{in}}^{2}(t)$.

In order to calculate $\sigma_{v_{\text {in }}^{2}}^{2}$, we need to express the power of the baseband components generated by $v_{\mathrm{in}}^{2}(t) \cdot v_{\mathrm{in}}^{2}(t)$ is centered on $\mathrm{dc}(b$ takes values in the range $\{-0.5 N, \ldots, 0.5 N\})$ and it is worth noting that phase noise is null in those IMD terms. We define the set of baseband IMD products generated by $v_{\text {in }}^{2}(t)$ as $K(b, N)$ and use the generating-function method to calculate its size $|K|$

$$
|K|= \begin{cases}N-1-|b|, & |b| \in\{1, \ldots, 0.5 N\} \\ N, & b=0 .\end{cases}
$$

The components in $K(b, N)$ add in power, except for $b=0$, in which case, they are all in-phase and add in amplitude. The distortion power $P_{K}(b, N)$ in the set $K$ is

$P_{K}(b, N)= \begin{cases}\frac{1}{8}(N-1-|b|)\left\langle z^{2}\right\rangle^{2}, & |b| \in\{1, \ldots, 0.5 N\} \\ \frac{1}{8}\left(N\left\langle z^{2}\right\rangle^{2}+2\left(\begin{array}{c}N \\ 2\end{array}\right)\left\langle z^{4}\right\rangle\right), & b=0 .\end{cases}$

Therefore, the variance $\sigma_{v_{\text {in }}^{2}}^{2}$ is expressed as

$$
\begin{aligned}
\sigma_{v_{\text {in }}^{2}}^{2} & =\sum_{b=-0.5 N}^{0.5 N} P_{K}(b, N) \\
& =\frac{1}{16} N\left\langle z^{2}\right\rangle^{2}\left(\frac{3}{2} N-1\right)+\frac{1}{8} N\left\langle z^{4}\right\rangle(N-1) .
\end{aligned}
$$

By substitution of (21) in (18), we get

$$
\begin{aligned}
P_{0}=a_{1}^{2} N_{0} \frac{\omega_{u}}{2 \pi} & +\frac{9}{16} a_{3}^{2} N_{0} \frac{\omega_{u}}{2 \pi} N \\
& \times\left[\left\langle z^{2}\right\rangle^{2}\left(\frac{3}{2} N-1\right)+2\left\langle z^{4}\right\rangle(N-1)\right] .
\end{aligned}
$$

Finally, we need to take into account the nonlinear circuit internal noise sources. We assume there is a noise contribution $P_{N}$ per carrier so that the output carrier SNR can be expressed as

$$
\mathrm{SNR}=\frac{P_{c}(b, N)}{P_{\mathrm{IM}}(b, N)+\sigma_{\mathrm{CPE}}^{2}(b, N)+\sigma_{\mathrm{ICI}}^{2}(b, N)+P_{0}+P_{N}} .
$$

\section{SNR Mapping to SER and BER}

We have obtained an expression that maps the quantities $N$, $a_{1}, a_{3},\left\langle z^{2}\right\rangle$, and $b$ (the distance in carrier spacing from the lower edge of the OFDM channel) to the carrier SNR. Neglecting the effect of phase noise and setting $P_{0}=P_{N}=0$, we can isolate the effect of IMD distortion on the output SNR. In that case, the central limit theorem ensures that the distortion components can 


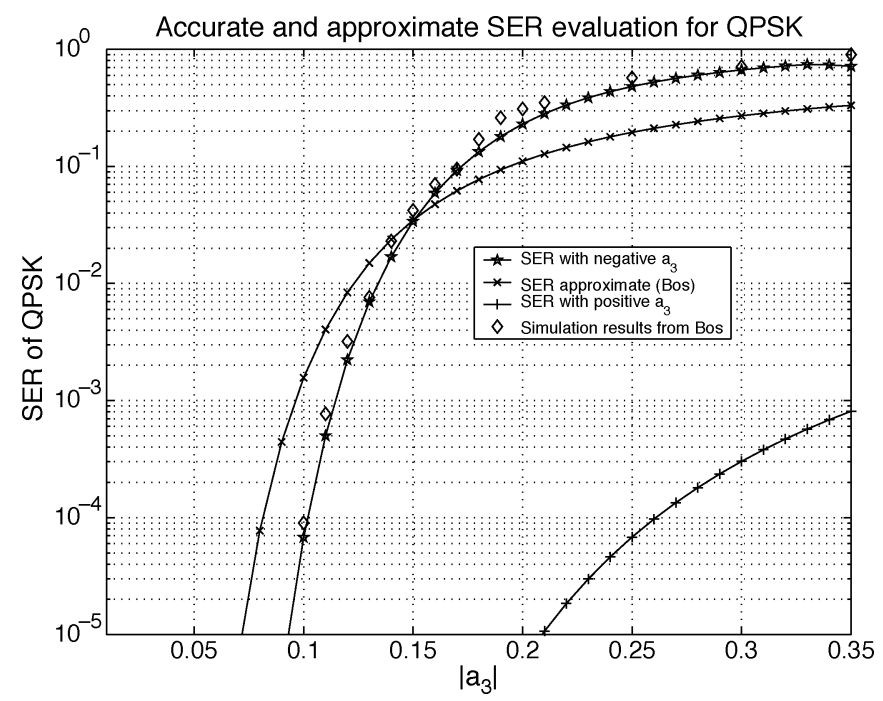

Fig. 2. Comparison of exact and approximate SER evaluation. QPSK OFDM, $N=1024, a_{1}=1$.

be approximated as Gaussian noise. We can as a result relate the carrier SNR to average SER for $M$-QAM and BER for DQPSK through the formulas provided in [27]. For the SER, we will use the formula

$$
\mathrm{SER}=1-\left(1-2\left(1-\frac{1}{\sqrt{M}}\right) Q\left(\sqrt{\frac{3 \mathrm{SNR}_{s}}{M-1}}\right)\right)^{2}
$$

where $\mathrm{SNR}_{s}$ is the symbol SNR, $M$ is the number of constellation points of the $M$-QAM, $Q(\cdot)=0.5 \operatorname{erfc}(\cdot / \sqrt{2})$ with $\operatorname{erfc}(\cdot)$ being the complementary error function.

For the DQPSK BER, we have the expression

$$
\mathrm{BER}=Q_{1}(a, b)-\frac{1}{2} I_{0}(a \cdot b) e^{-\frac{a^{2}+b^{2}}{2}}
$$

where $Q_{1}(\cdot)$ is Marcum's $Q$ function defined as

$$
Q_{1}(a, b)=e^{-\frac{a^{2}+b^{2}}{2}} \sum_{n=0}^{\infty}\left(\frac{a}{b}\right)^{n} I_{n}(a \cdot b)
$$

with $(a, b)=\left(\sqrt{\operatorname{SNR}_{b}(2-\sqrt{2})}, \sqrt{\operatorname{SNR}_{b}(2+\sqrt{2})}\right), \operatorname{SNR}_{b}$ is the bit SNR, $\mathrm{SNR}_{b}=\mathrm{SNR}_{s} / 2$. Finally, $I_{n}(\cdot)$ is the modified Bessel function of the first kind and order $n$.

In Fig. 2, we compare the results of our analysis to: 1) the approximate results in [12] denoted in the graph as "SER approximate (Bos)" and 2) the simulated results in [12] denoted in the graph as "Simulation results from Bos." In the same graph, we plot the SER evaluated for QPSK OFDM with $N=1024$ and unit input power, $0.5\left\langle z^{2}\right\rangle N=1$ both for negative $a_{3}$ (denoted as "SER with negative $a_{3}$ ") and positive $a_{3}$ (denoted as "SER with positive $a_{3}$ "), following our accurate SNR estimator. We note that the results produced by our accurate SNR estimator match very well with the simulated results presented in [12], thus offering a degree of validation. Our exact analysis substantially increases the degree of accuracy in SER estimation, as

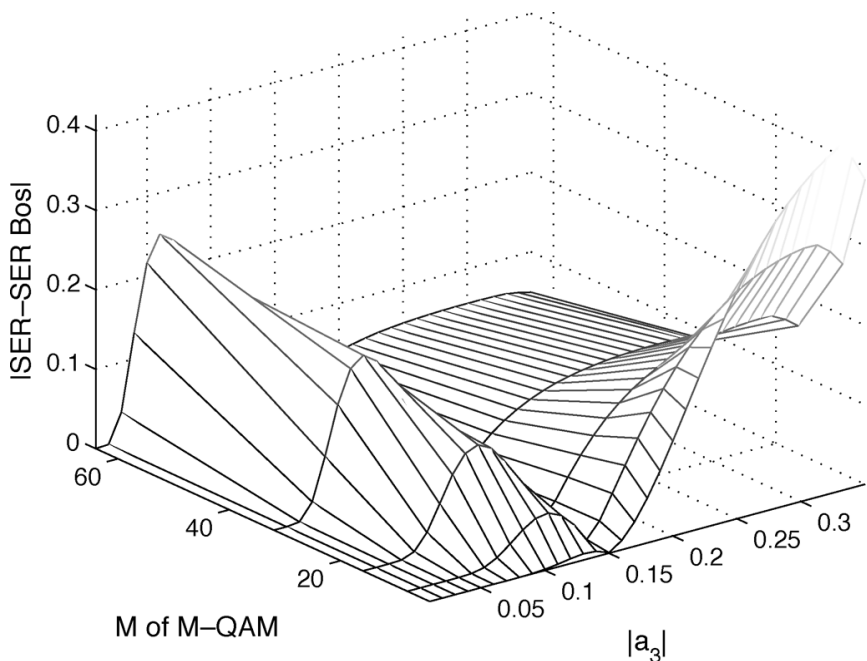

Fig. 3. Absolute value of the difference between the exact and approximate SER expressions as a function of the number of constellation points $M$ and $\left|a_{3}\right|$. We have set $N=1024$ and $a_{1}=1$.

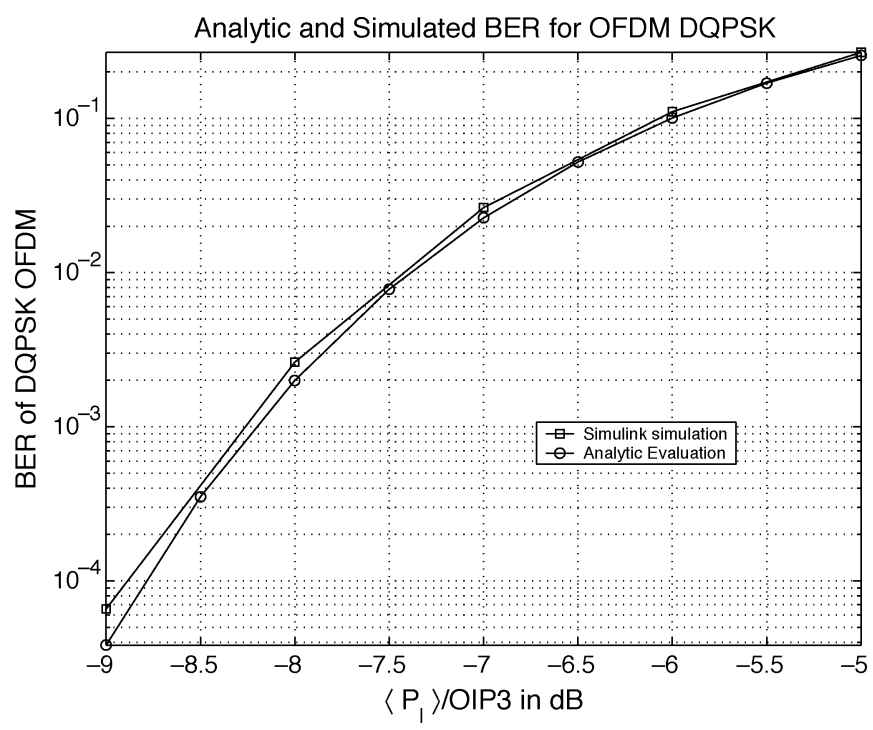

Fig. 4. Analytic and simulation BER for DQPSK OFDM, $N=1536$, $\omega_{u}=2 \pi \mathrm{krad} / \mathrm{s}$, OIP $3=8 \mathrm{dBm}$.

compared to the approximate, in the case of negative $a_{3}$. Moreover, in the case of positive $a_{3}$, the approximate analysis does not account for the gain enhancement and the approximate SER prediction is rather pessimistic.

In Fig. 3, we plot the absolute value of the difference between the exact and approximate SER expressions as a function of the number of constellation points and of the absolute value of the nonlinearity coefficient $\left|a_{3}\right|$, assuming $a_{3}<0$. The input power is unit, $N=1024$ and $a_{1}=1$. The underestimation or overestimation of the SER is shown to be more important for constellations with either a small or a large number of constellation points. Therefore, in such OFDM systems, the exact SNR expressions should be used in IMD performance analysis.

Furthermore, in Fig. 4, we compare BER results obtained from our analysis in the case of negative $a_{3}$ to simulation results 


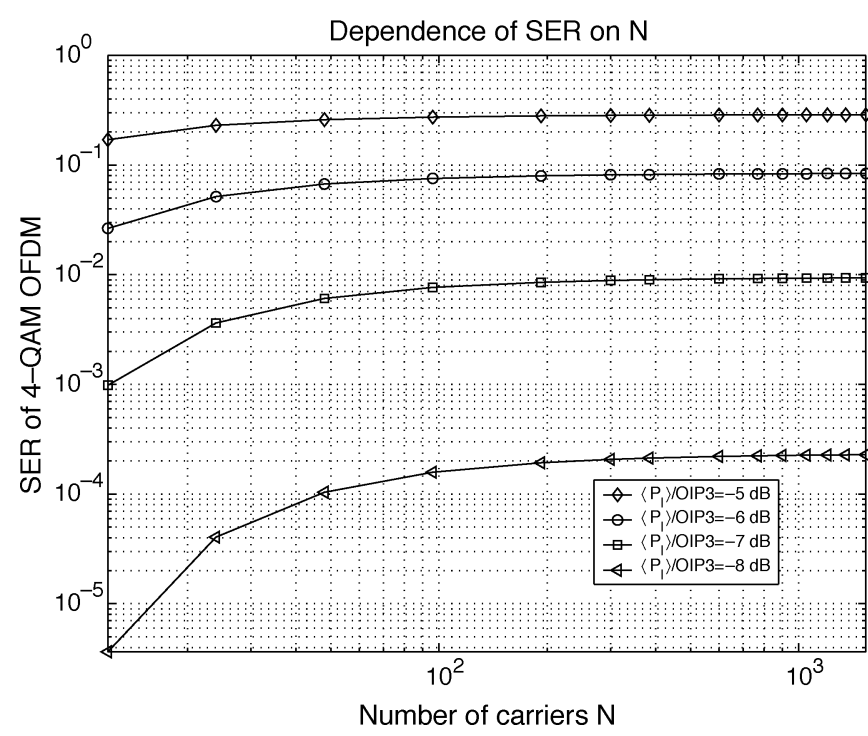

Fig. 5. SER of 4-QAM for different values of $\left\langle P_{l}\right\rangle /$ OIP 3 versus the number of carriers.

obtained from a DAB OFDM simulator developed in Simulink/ MATLAB. The output BER was evaluated for DQPSK OFDM over 100 symbols in our simulator. The OIP3 was set to $8 \mathrm{dBm}$, while the linear gain was assumed unit $a_{1}=1$ and the simulator generated $N=1536$ carriers at a carrier spacing $\omega_{u}=$ $2 \pi \mathrm{krad} / \mathrm{s}$. The results present good match and further validate our analysis.

To continue, we define as "linear output power" the output power in the absence of nonlinearities $\left(a_{3}=0\right)$

$$
\left\langle P_{l}\right\rangle=\frac{1}{2} N a_{1}^{2}\left\langle z^{2}\right\rangle
$$

and plot in Fig. 5 the SER of a 4-QAM OFDM versus the number of carriers for different values of $\left\langle P_{l}\right\rangle / \mathrm{OIP} 3$. For sufficiently high values of $N$ (around 100), the SER depends only on the ratio of the linear term to the OIP3. For values of $N$ below 100, the SER is slightly improved. It is thus inferred that the proportion of correlated and uncorrelated to the carrier intermodulation terms tends to be insensitive to the OFDM service layout. This result is in accordance with (9) and (10) as the dominant terms in both expressions are of the order of $\left(N^{2}\right)$ so that their ratio is asymptotically independent of $N$.

Finally, in Figs. 6 and 7, we investigate the performance of OFDM with $N=96$ for different constellations versus $\left\langle P_{l}\right\rangle / \mathrm{OIP} 3$. As long as the target BER in the case of DQPSK or SER for $M$-QAM is defined, we can identify the requirement on $\left\langle P_{l}\right\rangle /$ OIP3. From Fig. 7 stems that a decrease of approximately $1.4 \mathrm{~dB}$ in $\left\langle P_{l}\right\rangle / \mathrm{OIP} 3$ is necessary for each doubling of the number of constellation points. This arises because, for large $M$, the SER is approximately a function of SNR/ $M$ [27] and, thus, a doubling of $M$ may be compensated by a 3-dB increase in SNR. From (10), it follows that the SNR is proportional to $a_{3}^{-2}$ or, equivalently, from (2) to $\mathrm{OIP}^{2}$.

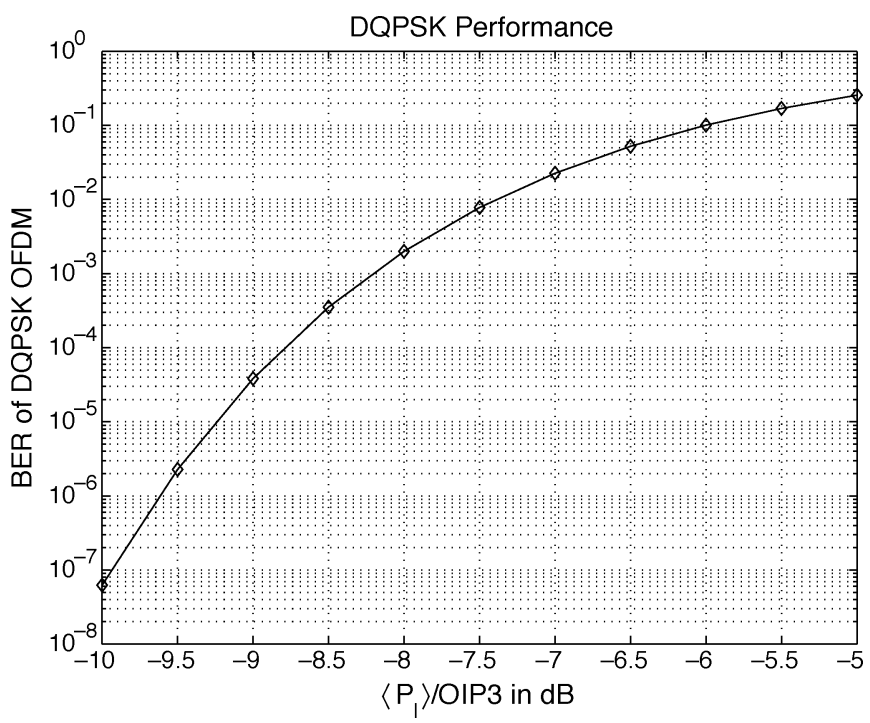

Fig. 6. BER of DQPSK OFDM with $N=96$ versus $\left\langle P_{l}\right\rangle / \mathrm{OIP} 3$.

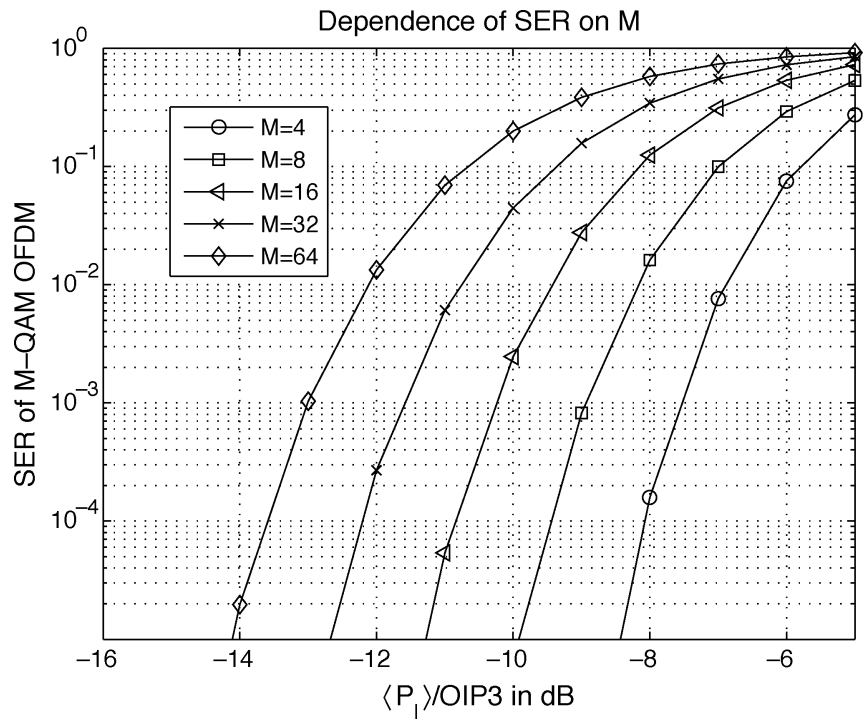

Fig. 7. SER of $M$-QAM OFDM with $N=96$ versus $\left\langle P_{l}\right\rangle /$ OIP3.

\section{OFDM With AN ADJACENT ChanNel OF DIFFERENT AMPLITUDE}

\section{A. Adjacent Channel at Integer Spacing}

In Section III, we examined the performance of an OFDM service under third-order nonlinearities. Here, we will further assume an adjacent OFDM channel of different amplitude. The adjacent channel amplitude relative to the carrier is $H_{\text {adj }}$, and $g$ is the spacing between adjacent channels, in units of carrier spacing. The input signal is expressed as

$$
\begin{aligned}
v_{\text {in }}(t)= & \sum_{\substack{k=0 \\
k \neq \frac{N}{2}}}^{N} \Re\left(z_{k} e^{j\left[\left(\omega_{d}+k \omega_{u}\right) t+\theta_{k}\right]}\right) \\
& +H_{\text {adj }} \sum_{\substack{k=N+g \\
k \neq N+g+\frac{N}{2}}}^{2 N+g} \Re\left(z_{k} e^{j\left[\left(\omega_{d}+k \omega_{u}\right) t+\theta_{k}\right]}\right) .
\end{aligned}
$$


TABLE III

Sub-SubSETS ACCORDING RELATIVE TO THEIR CARRIER AMPLITUDE

\begin{tabular}{cccc}
\hline 1 & $H_{a d j}$ & $H_{a d j}{ }^{2}$ & $H_{a d j}{ }^{3}$ \\
\hline \hline$T_{10}$ & $T_{11}$ & $T_{12}$ & $T_{13}$ \\
$T_{20}$ & $T_{21}$ & $T_{22}$ & $T_{23}$ \\
$T_{30}$ & $T_{31}$ & $T_{32}$ & $T_{33}$ \\
$T_{40}$ & $T_{41}$ & $T_{42}$ & $T_{43}$ \\
\hline
\end{tabular}

In order to calculate the size $|U|$ of the set $U(b, N)$ of in-band IMD products, we identify the generating function for this problem as

$$
\begin{aligned}
G(x)= & \left(P_{x}+H_{\mathrm{adj}} x^{(N+g)} P_{x}\right)^{2} \\
& \times\left(P_{-x}+H_{\mathrm{adj}} x^{-(N+g)} P_{-x}\right) \\
= & x^{-(2 N+g)}\left(1+H_{\mathrm{adj}} x^{(N+g)}\right)^{2} \\
& \times\left(H_{\mathrm{adj}}+x^{(N+g)}\right)\left(\frac{1-x^{(N+1)}}{1-x}-x^{\frac{N}{2}}\right)^{3}
\end{aligned}
$$

where

$$
\begin{aligned}
P_{x} & =1+x+\ldots+x^{N}-x^{\frac{N}{2}} \\
P_{-x} & =1+x^{-1}+\cdots+x^{-N}-x^{-\frac{N}{2}} .
\end{aligned}
$$

At present, we have assumed that $g$ is a multiple of the carrier spacing to simplify calculations. Later on, we will disengage from that requirement and examine the case of a noninteger inter-channel spacing.

The case presenting the greatest interest is that of an immediate adjacent channel, where normally $g<N$. The size $|U|$ of the set $U(b, N)$ of in-band IMD products is expressed in (61), shown at the bottom of page 3313 when $2<g<0.25 N$.

To get the output SNR, we have to identify the subsets $T_{1}, T_{2}$, $T_{3}$, and $T_{4}$, as defined in Table II. However, within each of these subsets, we have to further identify sub-subsets of amplitudes. Use of the generating-function technique provides the sizes of the sub-subsets defined in Table III. All amplitudes are relative to the carrier amplitude.

The sizes of the various sub-subsets are calculated in Appendix II.

The useful in-band output power is thus expressed as

$$
\begin{aligned}
P_{c}(b, g, N)=\left\langle\frac { 1 } { 2 } \left[ a_{1} z_{b}+\frac{3}{4} a_{3} z_{b}^{3}+\frac{3}{2} a_{3} z_{b} \sum_{i=1}^{\left|T_{3_{0}}\right|} z_{l, i}^{2}\right.\right. \\
\left.\left.\quad+\frac{3}{2} a_{3} H_{\mathrm{adj}}^{2} z_{b} \sum_{i=1}^{\left|T_{3_{2}}\right|} z_{m, i}^{2}\right]^{2}\right\rangle \\
=\frac{1}{2}\left[\begin{array}{c}
a_{1}^{2}\left\langle z^{2}\right\rangle+\frac{9}{16} a_{3}^{2}\left\langle z^{6}\right\rangle \\
+\frac{9}{4} a_{3}^{2}\left(\left|T_{3_{0}}\right|+H_{\mathrm{adj}}^{4}\left|T_{3_{2}}\right|\right)\left\langle z^{2}\right\rangle\left\langle z^{4}\right\rangle \\
+\frac{9}{2} a_{3}^{2}\left(\left(\begin{array}{c}
\left|T_{3_{0}}\right| \\
2
\end{array}\right)+H_{\mathrm{adj}}^{4}\left(\begin{array}{c}
\left|T_{3_{2}}\right| \\
2
\end{array}\right)\right)\left\langle z^{2}\right\rangle^{3}
\end{array}\right.
\end{aligned}
$$

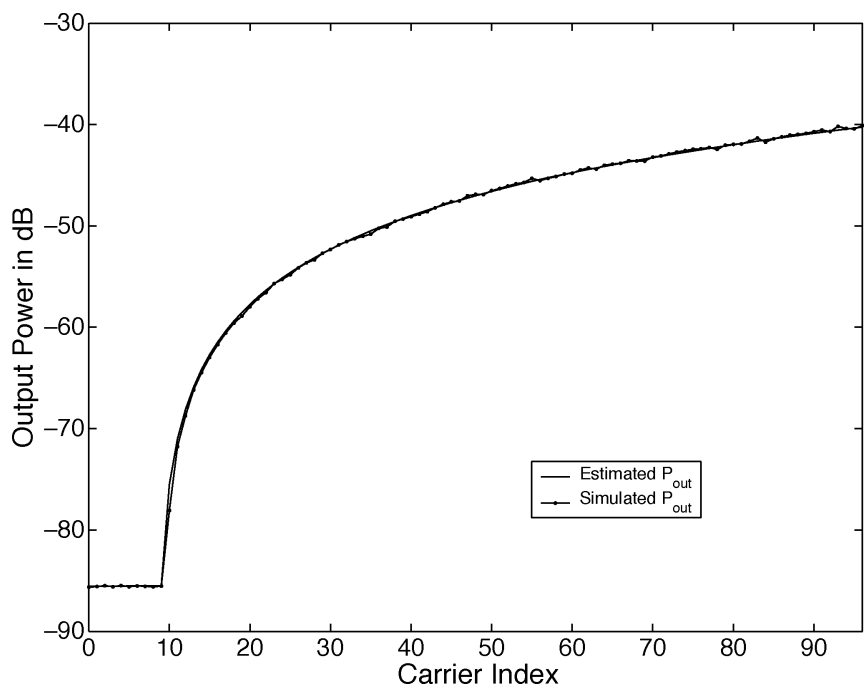

Fig. 8. Overall in-band output power of a QPSK OFDM channel with an adjacent channel $60 \mathrm{~dB}$ higher. $N=96, g=10,\left\langle P_{l}\right\rangle=-30 \mathrm{dBm}, \mathrm{OIP} 3=$ $18.24 \mathrm{dBm}$.

$$
\begin{aligned}
& +\frac{3}{2} a_{1} a_{3}\left\langle z^{4}\right\rangle \\
& +3 a_{1} a_{3}\left(\left|T_{3_{0}}\right|+H_{\text {adj }}^{2}\left|T_{3_{2}}\right|\right)\left\langle z^{2}\right\rangle^{2} \\
& +\frac{9}{4} a_{3}^{2}\left(\left|T_{3_{0}}\right|+H_{\text {adj }}^{2}\left|T_{3_{2}}\right|\right)\left\langle z^{2}\right\rangle\left\langle z^{4}\right\rangle \\
& \left.+\frac{9}{2} a_{3}^{2} H_{\text {adj }}^{2}\left|T_{3_{0}}\right|\left|T_{3_{2}}\right|\left\langle z^{2}\right\rangle^{3}\right] .
\end{aligned}
$$

With respect to intermodulation noise, using similar reasoning to the case of a single OFDM channel, the various sub-subsets of subsets $T_{2}$ and $T_{4}$ are mutually uncorrelated and resulting cross-terms have null averages. Therefore, all sub-subsets add in power. The corresponding intermodulation noise is now expressed as

$$
\begin{aligned}
& P_{\mathrm{IM}}(b, g, N) \\
& \begin{array}{r}
=\frac{9}{32} a_{3}^{2}\left\langle z^{2}\right\rangle\left[\left(\left|T_{2_{0}}\right|+H_{\text {adj }}^{2}\left|T_{2_{1}}\right|+H_{\text {adj }}^{4}\left|T_{2_{2}}\right|\right)\left\langle z^{4}\right\rangle\right. \\
+4
\end{array} \\
& +\left|T_{4_{0}}\right|+H_{\text {adj }}^{2}\left|T_{4_{1}}\right|+H_{\text {adj }}^{2}\left|T_{4_{2}}\right| \\
& \left.\left.+H_{\text {adj }}^{6}\left|T_{4_{3}}\right|\right)\left\langle z^{2}\right\rangle^{2}\right] .
\end{aligned}
$$

In Fig. 8 , we plot the total output power $P_{\text {out }}=P_{c}+P_{\mathrm{IM}}$ and compare the results of our estimation with simulation results averaged over 1000 symbols. The analytic and simulation curves are practically indistinguishable. The OFDM channel has $N=96$ carriers, the adjacent channel is at a distance of $g=10$ carriers, the expected value of the output linear power, as expressed in (26), is $\left\langle P_{l}\right\rangle=-30 \mathrm{dBm}$ and OIP3 $=18.24 \mathrm{dBm}$.

In the specific example, the adjacent channel is chosen $H_{\text {adj }}=60 \mathrm{~dB}$ higher than the OFDM channel of interest. As expected from a closer inspection of (71)-(73), shown at the bottom of page 3314, the output power on carriers with indices 0 to $(g-1)$ contains intermodulation noise from set $T_{4}$ with relative power 0,60 , and $120 \mathrm{~dB}$. From (74), shown at the bottom of page 3314 , it becomes clear that IMD products purely generated from intermodulation of the adjacent channel 


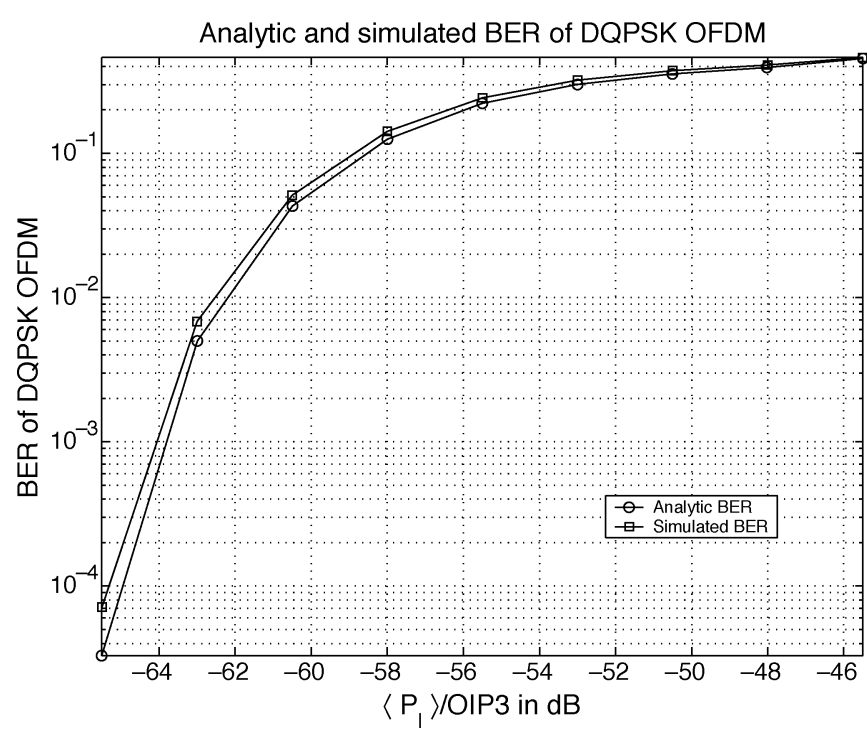

Fig. 9. Simulated versus analytic BER for DQPSK BER with an adjacent channel $40 \mathrm{~dB}$ higher than the channel of interest. $N=1536, g=200$, $\omega_{u}=2 \pi \mathrm{krad} / \mathrm{s}$

with itself and of relative power $180 \mathrm{~dB}$ affect only carriers that are at least $g=10$ carriers away from the lower edge of the OFDM channel. We conclude that, in the presence of a significantly higher immediate adjacent channel, OFDM carriers in its vicinity are much more severely affected.

The carrier SNR is expressed as

$$
\mathrm{SNR}=\frac{P_{c}(b, g, N)}{P_{\mathrm{IM}}(b, g, N)+P_{N}}
$$

where $P_{c}$ is given in (31), $P_{\mathrm{IM}}$ is expressed in (32), and $P_{N}$ is the circuit noise. The estimation of BER for DQPSK OFDM and SER for $M$-QAM OFDM are produced based on (24) and (25).

The term $P_{\mathrm{IM}}$ contains IMD products of the adjacent channel with itself, thus we expect a severe degradation of the carrier SNR in the presence of a high adjacent channel compared to the case of single OFDM channel through similar nonlinear circuits. The result is a reduction in the effective dynamic range of the OFDM receiver. It is thus inferred that, in the receiver downconversion chain, either channel selection has to be performed at an early stage or a strict requirement on RF circuits linearity has to be adopted.

In order to validate the derived SNR expression, in Fig. 9 we compare simulation results using the Simulink/MATLAB simulator with the estimated BER based on (31)-(33). The DQPSK OFDM simulator generates $N=1536$ carriers, the inter-channel spacing is $g=200$, and the carrier spacing is $\omega_{u}=2 \pi \mathrm{krad} / \mathrm{s}$ while the adjacent channel is at $H_{\text {adj }}=40 \mathrm{~dB}$. Theoretical and simulation results match well.

In Figs. 10 and 11, we investigate the impact of an adjacent channel on the SER of an $M$-QAM OFDM. In Fig. 10, we have $N=96, g=12$ and an adjacent channel of relative amplitude $H_{\text {adj }}=20 \mathrm{~dB}$ and highlight the impact of the number of constellation points $M$ on the output SER. Our previous remark

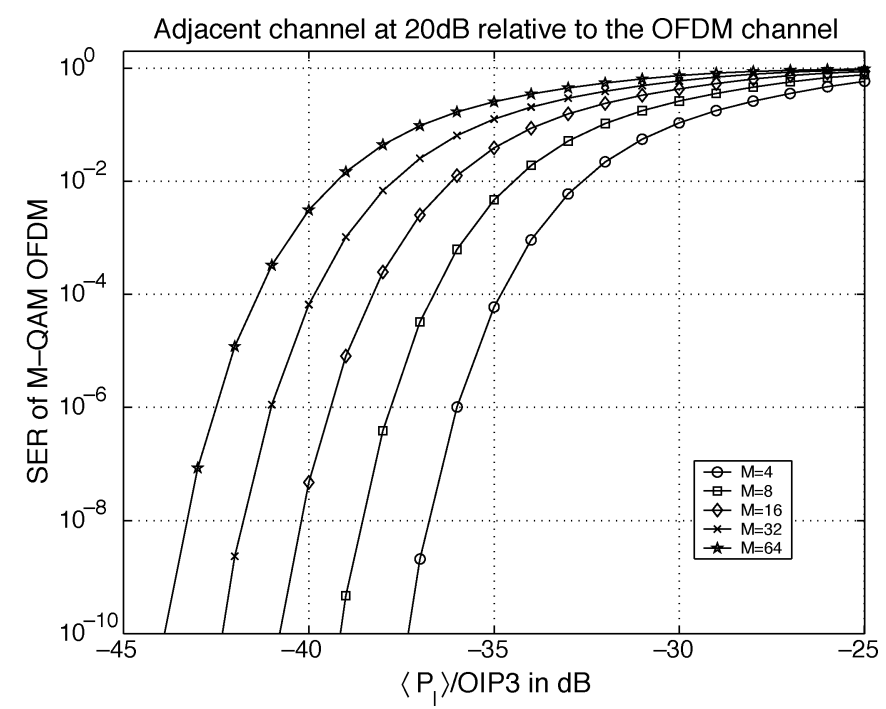

Fig. 10. SER versus $\left\langle P_{l}\right\rangle /$ OIP 3 for $M$-QAM OFDM with $N=96$ and $g=$ 12 . The relative power of the adjacent channel is $20 \mathrm{~dB}$.

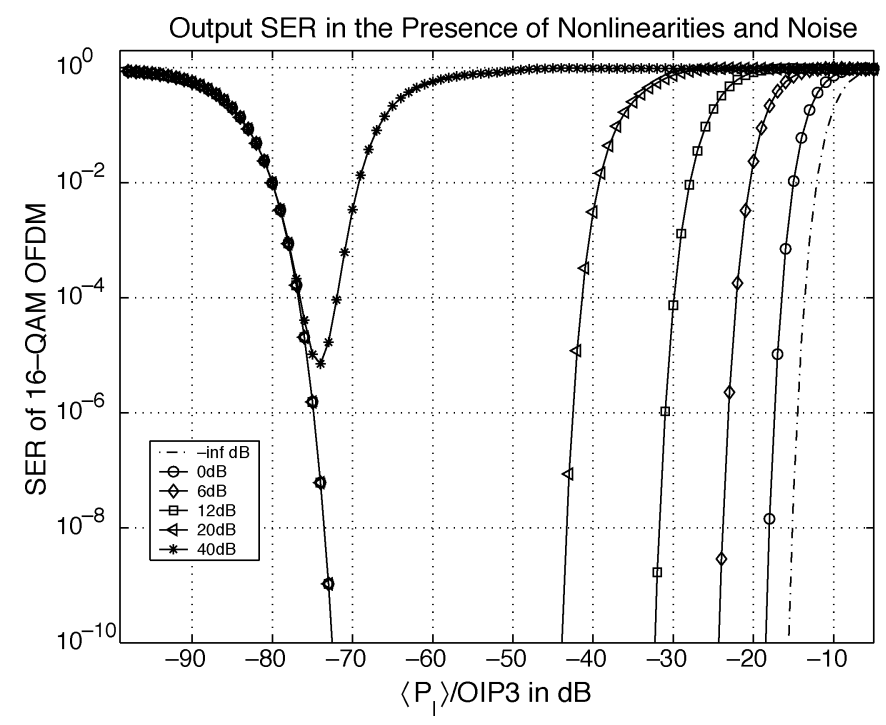

Fig. 11. SER versus $\left\langle P_{l}\right\rangle /$ OIP 3 for 16-QAM OFDM with $N=1536, \omega=$ $2 \pi \mathrm{krad} / \mathrm{s}, g=200$, and various adjacent channel relative amplitudes.

about the reduction in receiver dynamic range is confirmed. In order to achieve a target SER for a given constellation, we need to decrease the nonlinear circuit output power level. The limitation in decreasing the output power is placed by the nonlinear circuit internal noise sources that at low output power levels will have a nonnegligible effect on the carrier SNR.

The effect is investigated in Fig. 11, where, we assume a circuit with noise figure $\mathrm{NF}=15 \mathrm{~dB}^{2}$ and plot the SER of 16-QAM OFDM with $N=1536, \omega=2 \pi \mathrm{krad} / \mathrm{s}$, and $g=200$ for various adjacent channel power levels. From this graph, we can identify in each case the optimal reception region in regard to the ratio $\left\langle P_{l}\right\rangle / \mathrm{OIP} 3$. As long as the circuit operates in that region, the system SER can be kept under the occasional target. When $H_{\text {adj }}$ is large, (32) is dominated by the term in $H_{\text {adj }}^{3}$ and

\footnotetext{
${ }^{2} \mathrm{An} \mathrm{NF}=15 \mathrm{~dB}$ is a pessimistic value used for illustrative purposes
} 
for every 20-dB increase in adjacent channel power, a 30-dB decrease in $\left\langle P_{l}\right\rangle / \mathrm{OIP} 3$ is necessary to prevent performance degradation. This follows from (2) and the quadratic dependency of the noise on $a_{3}$.

As an interesting limiting case, when the adjacent channel amplitude becomes $H_{\text {adj }}=40 \mathrm{~dB}$ higher than the OFDM channel of interest, we can identify the optimal value of the quantity $\left\langle P_{l}\right\rangle / \mathrm{OIP} 3$ in order to minimize the SER. In the receiver design, extra care should be put into meeting the compromise between circuit NF and nonlinearities, as well as in the detection of adjacent channels and the adjustment of automatic gain control (AGC) stages accordingly.

As a concluding comment, previous analyses of the effects of adjacent channel on multicarrier systems [19], [29] were based on the evaluation of the spectral regrowth of the adjacent channel alone. According to the terminology we introduced, this implies that only the subset $T_{4_{3}}$ was taken into account. There is an underestimation of the adjacent channel effect, as subsets $T_{1}, T_{2}, T_{3}, T_{4_{0}}, T_{4_{1}}$, and $T_{4_{2}}$ were completely disregarded. Unless the joint effect of the channel of interest and of the adjacent is investigated, the estimation of the intermodulation power is underestimated.

\section{B. Adjacent Channel at Noninteger Spacing}

We will now consider the case of an adjacent channel at a noninteger spacing in units of OFDM carrier spacing $\omega_{u}$ so $g$ is now a rational number instead of an integer. The issue is to identify which of the previously defined subsets fall exactly on carrier frequencies in the band of interest or in between two carriers. We express $g=g^{\prime}+$ offset, where $g^{\prime}=\lfloor g\rfloor$ and offset $=\operatorname{rem}\left(g / g^{\prime}\right), \operatorname{rem}(\cdot)$ being the remainder of the integer division. We examine the various subsets separately.

Subsets $T_{1_{0}}, T_{2_{0}}, T_{3_{0}}$, and $T_{4_{0}}$ are generated from combinations of the channel of interest and fall on carrier frequencies.

Subsets $T_{1_{1}}, T_{2_{1}}, T_{3_{1}}$, and $T_{4_{1}}$ are generated from combinations of two carriers of the wanted channel and one carrier from the adjacent. The IMD products in the above sets fall on frequencies $\omega_{b}=\omega_{b}^{\prime}$ - offset, where $\omega_{b}^{\prime}$ is the frequency the products would fall on if the adjacent channel was at a spacing $g^{\prime}$.

Subsets $T_{1_{2}}, T_{2_{2}}, T_{3_{2}}$, and $T_{4_{2}}$ are generated from the combination of two carriers of the adjacent channel and one of the wanted and fall on frequencies $\omega_{b}=\omega_{b}^{\prime}+$ offset - offset $=\omega_{b}^{\prime}$. Therefore, these sets fall exactly on the frequencies defined in the analysis involving an adjacent channel at an integer spacing.

Subset $T_{4_{3}}$ is generated exclusively from combinations of carriers of the adjacent channel and the IMD products fall on frequencies $\omega_{b}=\omega_{b}^{\prime}+$ offset + offset - offset $=\omega_{b}^{\prime}+$ offset. Therefore, all IMD products in this set fall on the frequencies defined from the previous analysis plus offset.

The IMD products that fall in between two carriers affect all the OFDM carriers to a varying extend. This phenomenon is the result of the time windowing used to isolate individual OFDM symbols. In the following, we assume a rectangular time window of length $2 \pi / \omega_{u}$ is used before the ADC converter and the subsequent FFT. In the frequency domain, this is equivalent to convolving the power spectrum with a $\operatorname{sinc}^{2}\left(\omega / \omega_{u}\right)$. The overall effect of the time windowing of the sampler of the ADC and of the FFT block is to project part of the in-band IMD distortion power onto all OFDM carriers. The projected IMD power onto an individual carrier is scaled by

$$
M_{b}=\operatorname{sinc}^{2}\left(\frac{\omega_{b}}{\omega_{u}}\right)=\operatorname{sinc}^{2}\left(\frac{\omega_{b^{\prime}}}{\omega_{u}}+\text { offset }\right) .
$$

The intermodulation noise power on a carrier at a distance $b \cdot \omega_{u}$ from the lower edge of the OFDM band is expressed as

$$
\begin{aligned}
& P_{\mathrm{IM} o f}(b, g, N, \text { offset }) \\
& =\frac{9}{32} a_{3}^{2}\left\langle z^{2}\right\rangle\left[\left|T_{2_{0}}(b)\right|\left\langle z^{4}\right\rangle+4\left|T_{4_{0}}(b)\right|\left\langle z^{2}\right\rangle^{2}\right. \\
& +H_{\text {adj }} \sum_{\substack{m=-b, m \neq \frac{N}{2}-b}}^{N-b}\left(\left|T_{2_{1}}(q)\right|\left\langle z^{4}\right\rangle+4\left|T_{4_{1}}(q)\right|\right) \\
& \times\left\langle z^{2}\right\rangle^{2} \operatorname{sinc}^{2}\left(m_{1}\right) \\
& +H_{\text {adj }}^{2}\left(\left|T_{2_{2}}(b)\right|\left\langle z^{4}\right\rangle+4\left|T_{4_{2}}\right|(b)\left\langle z^{2}\right\rangle^{2}\right) \\
& \left.+4 H_{\mathrm{adj}}^{3} \sum_{\substack{m=-b \\
m \neq \frac{N}{2}-b}}^{N-b}\left|T_{4_{3}}(q)\right|\left\langle z^{2}\right\rangle^{2} \operatorname{sinc}^{2}\left(m_{2}\right)\right]
\end{aligned}
$$

where

$$
\begin{aligned}
m_{1} & =m-\text { offset } \\
m_{2} & =m+\text { offset } \\
q & =m+b .
\end{aligned}
$$

As $\left|T_{1_{1}}\right|=\left|T_{3_{1}}\right|=0$, the carrier power is not altered and the carrier SNR is expressed as

$$
\mathrm{SNR}=\frac{P_{c}(b, g, N)}{P_{\mathrm{IM}_{o f}}(b, g, N, \text { offset })+P_{N}}
$$

where $P_{c}$ is expressed in (31) and $P_{N}$ is the circuit noise. The overall intermodulation noise power is larger compared to the case of integer $g$ and OFDM will perform worse under a high power adjacent channel.

Validation of the presented SNR estimator is provided by comparison of the analytically evaluated BER for DQPSK OFDM and simulated results. The Simulink/MATLAB simulator generated $N=1536$ carriers and an adjacent channel at 40 $\mathrm{dB}$ higher at a frequency of $g=200.5$ with a carrier spacing of $\omega_{u}=2 \pi \mathrm{krad} / \mathrm{s}$. The results match well and are depicted in Fig. 12.

The SNR degradation strongly depends on the number of OFDM carriers. For a high adjacent channel, the main contribution in $P_{\mathrm{IM}_{o f}}$ comes from set $T_{4_{3}}$. For $b>g$, we can approx- 


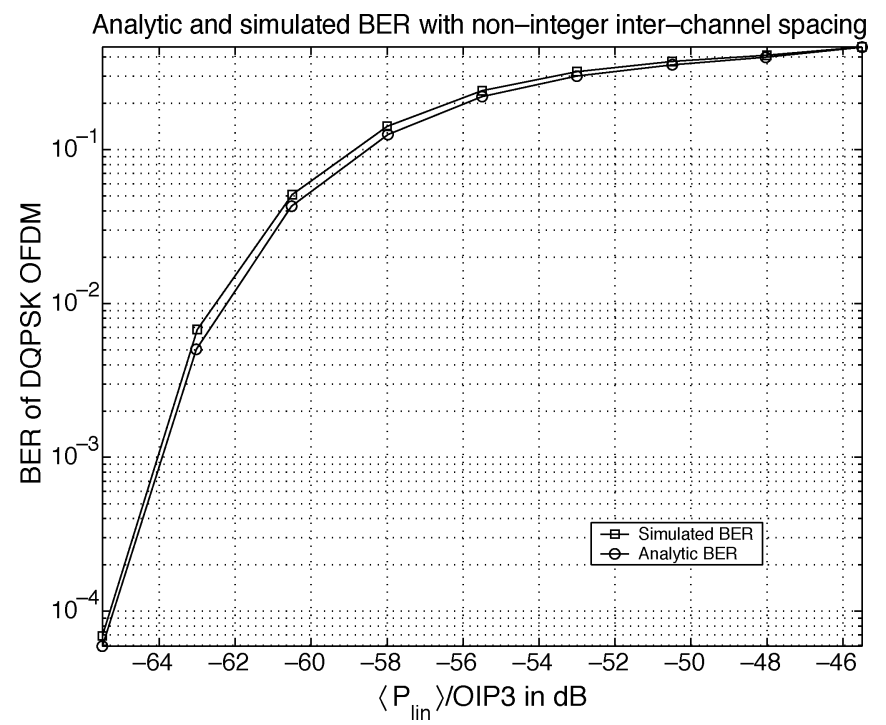

Fig. 12. Simulated versus analytic BER for DQPSK OFDM with an adjacent channel at $40 \mathrm{~dB}$ higher than wanted, at an offset of half the carrier spacing. $N=1536, g=200.5, \omega_{u}=2 \pi \mathrm{krad} / \mathrm{s}$.

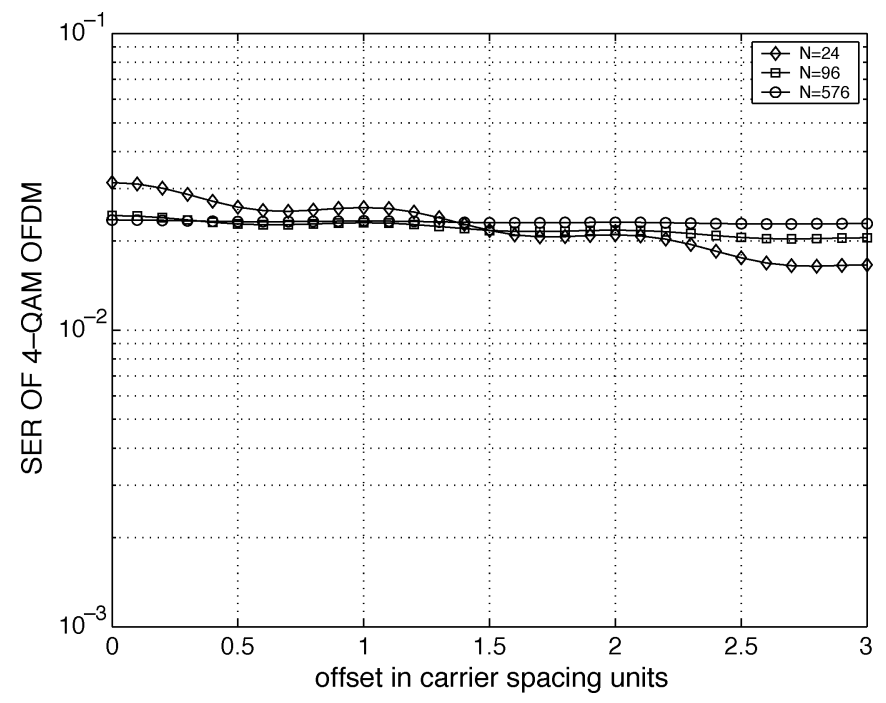

Fig. 13. SER of 4-QAM OFDM versus offset in adjacent channel spacing for OIP $3=18.24 \mathrm{dBm}$ and $H_{\text {adj }}=40 \mathrm{~dB}$.

imate the number of IMD as a constant $|T|$. Therefore, the final term in (35) can be approximated by

$4 H_{\text {adj }}^{3} \sum_{\substack{m=-b \\ m \neq \frac{N}{2}-b}}^{N-b}\left|T_{43}(q)\right|\left\langle z^{2}\right\rangle^{2} \sin c\left(m_{2}\right)^{2} \approx 4 H_{\mathrm{adj}}^{3}|T|\left\langle z^{2}\right\rangle^{2} \sum_{\substack{b=0 \\ b \neq \frac{N}{2}}}^{N} M_{b}$.

The expression in (40) is an increasing function that asymptotically reaches $4 H_{\text {adj }}^{3}\left\langle z^{2}\right\rangle^{2}|T|$ as $N \rightarrow \infty$.

In Fig. 13, we plot the output SER of a 4-QAM OFDM with an adjacent channel at a distance $g=[0.1 N],[\cdot]$ denoting the closest integer rounding function, while $H_{\mathrm{adj}}=40 \mathrm{~dB}$ and $a_{1}=$ $1, a_{3}=-0.05$ and overall input power $20 \mathrm{dBm}$. For a variety of values of $N$, the offset increases from 0 to $3 \omega_{u}$. Two conclusions can be drawn. Firstly, that the larger $N$, the less varied is the SER, as we previously predicted. Secondly, the local minima of the SER as a function of the offset are determined by two tendencies: the decrease in the SER as the inter-channel gap $g$ increases and the expression in (40) becomes maximal when the offset is half the carrier spacing.

From the previous analysis, we conclude that although theoretically there is a slight gain in carrier SNR if the system designer chooses an integer inter-channel spacing, the effect is almost imperceivable in realistic terms. As a result, the analysis for an adjacent channel at an integer spacing lends itself as a quite precise approximation for the case of noninteger spacing and can, therefore, safely be adopted as the general case.

\section{OFDM WITH AN ARBITRARY NUMBER OF ADJACENT CHANNELS}

Finally, in this section, we examine the case of an OFDM channel along with an arbitrary number of adjacent channels of the same amplitude. In the following, we have included the central carrier in order to avoid overcomplicating the derived expressions. The input signal to the nonlinear device can be expressed as

$$
v_{\text {in }}(t)=\sum_{m=0}^{r} \sum_{k=0}^{N} \Re\left(z_{k, m} e^{j\left(\left(\omega_{d}+[m(N+g)+k] \omega_{u}\right) t+\theta_{k, m}\right)}\right)
$$

where $r$ is the number of adjacent OFDM channels. The generating function to calculate the size $|U|$ of the set $U$ of in-band IMD products is

$$
\begin{aligned}
G(x)= & \left(Q_{x}+x^{N+g} Q_{x}+\cdots+x^{r(N+g)} Q_{x}\right)^{2} \\
& \left.\times\left(Q_{-x}+x^{-(N+g)} Q_{-x}+\cdots+x^{-r(N+g)} Q_{-x}\right)\right) \\
= & x^{-N-r(N+g)}\left(\frac{1-x^{N+1}}{1-x}\right)^{3}\left(\frac{1-x^{(N+g)(r+1)}}{1-x^{N+g}}\right)^{3} \\
= & x^{-N-r(N+g)}\left(1-x^{N+1}\right)^{3}\left(1-x^{(N+g)(r+1)}\right)^{3} R(x)
\end{aligned}
$$

where

$$
\begin{aligned}
Q_{x} & =1+x+x^{2}+\cdots+x^{N} \\
Q_{-x} & =1+x^{-1}+x^{-2}+\cdots+x^{-N} \\
R(x) & =\sum_{n=0}^{\infty} u_{n} x^{n}
\end{aligned}
$$

with

$$
u_{n} \doteq \sum_{l=0}^{t}\left(\begin{array}{c}
\tau \\
\tau-2
\end{array}\right) \cdot\left(\begin{array}{c}
\nu \\
\nu-2
\end{array}\right)
$$

Finally,

$$
\begin{aligned}
t & =\left\lfloor\frac{n}{N+g}\right\rfloor \\
\tau & =n-(t-l)(N+g)+2 \\
\nu & =t-l+2 .
\end{aligned}
$$

The size $|U|$ of the set $U(b, g, N, r)$ of the in-band components is given in Appendix III. 


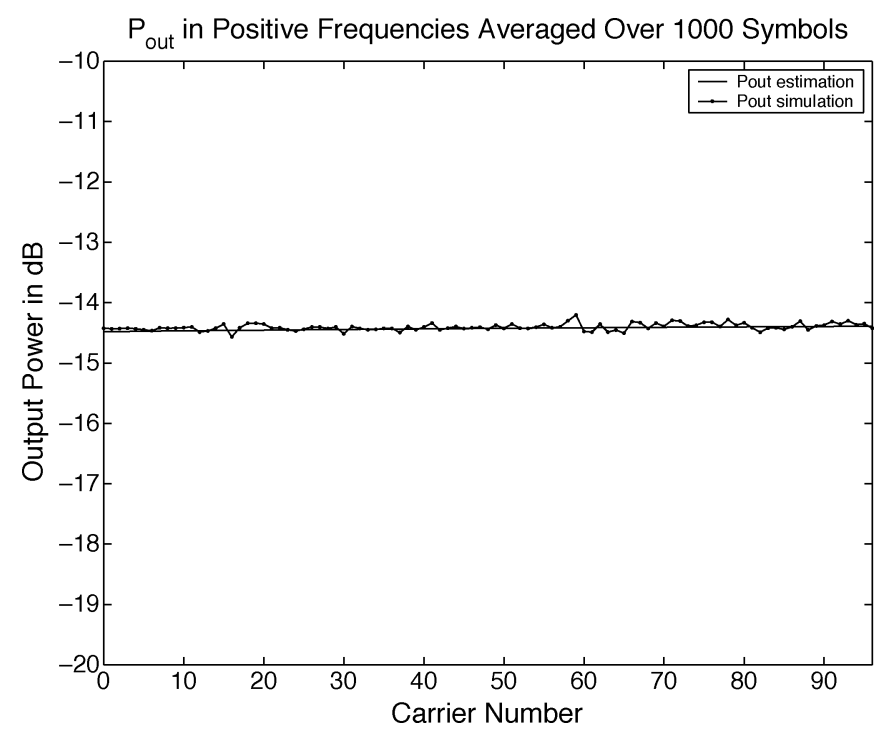

Fig. 14. Output power as estimated and through simulation.

Again, we will distinguish between the four subsets $T_{1}, T_{2}$, $T_{3}$, and $T_{4}$ whose phase relation is already described in Table II. The sizes of the relevant sets are calculated in Appendix III.

The useful power on a carrier is expressed as

$$
\begin{aligned}
P_{c}(b, g, N, r)=\frac{1}{2}[ & a_{1}^{2}\left\langle z^{2}\right\rangle+a_{3}^{2} \frac{9}{16}\left\langle z^{6}\right\rangle+a_{3}^{2} \frac{9}{4}\left|T_{3}\right|\left\langle z^{2}\right\rangle\left\langle z^{4}\right\rangle \\
& +a_{3}^{2} \frac{9}{2}\left(\begin{array}{c}
\left|T_{3}\right| \\
2
\end{array}\right)\left\langle z^{2}\right\rangle^{3}+a_{1} a_{3} \frac{3}{2}\left\langle z^{4}\right\rangle \\
& \left.+a_{3}^{2} \frac{9}{4}\left|T_{3}\right|\left\langle z^{2}\right\rangle\left\langle z^{4}\right\rangle+a_{1} a_{3} 3\left|T_{3}\right|\left\langle z^{2}\right\rangle^{2}\right] .
\end{aligned}
$$

The intermodulation noise results from subsets $T_{2}$ and $T_{4}$. Based on the fact that the cross-correlation terms have null averages, we express in closed form the intermodulation noise as

$$
P_{\mathrm{IM}}(b, g, N, r)=\frac{9}{32} a_{3}^{2}\left\langle z^{2}\right\rangle\left(\left|T_{2}\right|\left\langle z^{4}\right\rangle+4\left|T_{4}\right|\left\langle z^{2}\right\rangle^{2}\right) .
$$

In Fig. 14, we plot the estimated output power on a carrier $P_{\text {out }}=P_{c}+P_{\mathrm{IM}}$ and the simulated output power for QPSK OFDM with $N=96, g=12$, averaged over 1000 symbols. We have $r=4$ adjacent channels, and the overall linear output power

$$
\left\langle P_{l, r}\right\rangle=r \frac{1}{2} N\left(a_{1}\langle z\rangle\right)^{2}
$$

is $\left\langle P_{l, r}\right\rangle=36.02 \mathrm{dBm}$ and OIP3 $=18.24 \mathrm{dBm}$. The output power slightly increases with the carrier index.

The carrier SNR if we ignore all other noise sources is simply given by

$$
\mathrm{SNR}=\frac{P_{c}(b, g, N, r)}{P_{\mathrm{IM}}(b, g, N, r)} .
$$

In Fig. 15, we plot the SER of a 64-QAM OFDM as a function of $\left\langle P_{l, r}\right\rangle /$ OIP3 with and without adjacent channels. It is shown

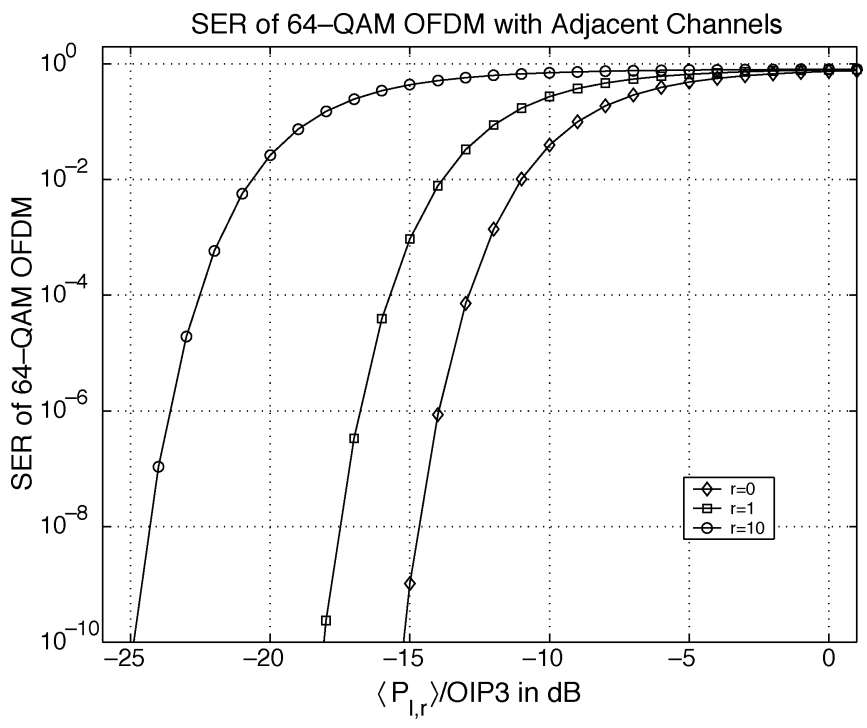

Fig. 15. Output SER of 64-QAM OFDM with $r$ adjacent channels.

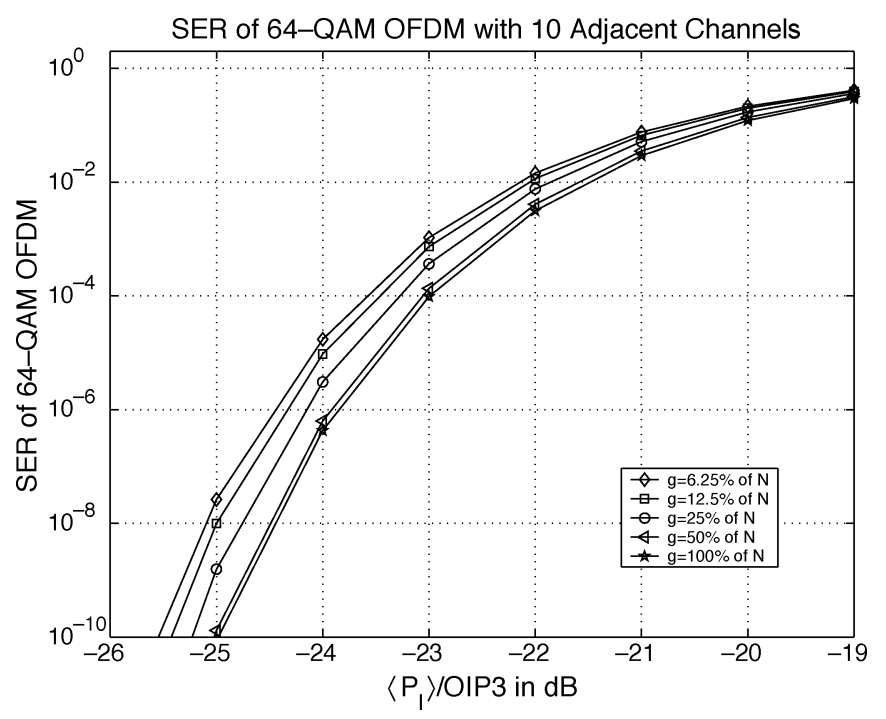

Fig. 16. SER of 64-QAM OFDM with ten adjacent channels as a function of the inter-channel spacing.

that for every $10-\mathrm{dB}$ increase in the total power of the adjacent channels, an approximately $10-\mathrm{dB}$ increase in the $\left\langle P_{l}\right\rangle / \mathrm{OIP} 3$ is necessary to maintain performance.

The effect of multiple adjacent channels can be mitigated by an increase of the inter-channel spacing. However, a limiting factor in the BER or SER improvement is placed by the fact that a number of IMD products is independent of $g$. The effect is investigated in Fig. 16 where we examine the effect of inter-channel spacing on the SER of a 64-QAM OFDM with ten adjacent channels. As the inter-channel spacing becomes equal to the OFDM channel bandwidth, the SER is still higher than in the case with no adjacent channels. Intermodulation products generated from mixing of the adjacent channels without the participation of the channel of interest places a limit on the achievable system performance. The effect should be taken into account, especially in the case of "congestive" broadcasting services. 


\section{CONCLUSIONS}

The use of combinatorics in nonlinear distortion analysis of OFDM signals can provide precise SNR evaluations. As long as the dominant circuit nonlinearities can be described by the theory of weakly nonlinear circuits, our SNR formulas are precise. We have derived in closed-form expressions for the carrier SNR in the cases of single OFDM channel, OFDM along with an adjacent channel of different amplitude and an OFDM channel with an arbitrary number of adjacent channels. A mapping to $M$-QAM SER and DQPSK BER was obtained by analyses of the occurring cross-term correlations. Comparison with other published studies shows that the new formulas notably increase the accuracy in SNR prediction [12].

Our results can provide important guidelines both to the OFDM service regulator and to the RF transceiver engineer and save valuable simulation time. Exploiting either a priori knowledge of the circuit nonlinear characteristics or an ad hoc estimation, our carrier SNR prediction can help improve the performance of subsequent soft decision stages of the decoder.

More importantly, the resilience of specific OFDM receiver architectures to nonlinearities can be precisely evaluated. The receiver designer can reliably achieve the necessary compromise between performing channel selection filtering at late stages of the tuner and using highly linear analog circuits.

Finally, our SNR estimators in the presence of single or multiple adjacent channels can help identify how the AGC levels should be chosen in order to avoid unacceptable levels of intermodulation noise.

\section{APPENDIX I}

\section{Set Sizes IN The CASE OF Single OFDM ChanNEL}

In the case of an OFDM channel through a third-order nonlinearity, we have calculated the sizes of sets $U, T_{1}, T_{2}, T_{3}$, and $T_{4}$ as follows:

$$
|U|= \begin{cases}-b^{2}+b N-3 b+0.5 N^{2}+1, & b \in B_{1} \\ 0.75 N^{2}-1.5 N, & b \in B_{2} \\ -b^{2}+b N+3 b+0.5 N^{2}-3 N+1, & b \in B_{3}\end{cases}
$$

where $B_{1}=\{0, \ldots, 0.5 N-1\}, B_{2}=\{0.5 N\}, B_{3}=\{0.5 N+$ $1, \ldots, N\}$

$$
\begin{aligned}
& \left|T_{1}\right|= \begin{cases}0, & b \in B_{2} \\
1, & b \in B_{1} \cup B_{3}\end{cases} \\
& \left|T_{2}\right|= \begin{cases}0.5\left(N-2+2 \mu_{1}\right), & b \in B_{4} \\
0.5\left(N-2-2 \mu_{1}\right), & b \in B_{5} \\
0.5\left(N-2-2 \mu_{2}+2 \mu_{3}\right) & b \in B_{6} \\
0.5\left(N-2 \mu_{1}\right), & b \in B_{7} \\
0.5\left(N-2+2 \mu_{1}\right), & b \in B_{8} \\
0.5\left(N-2-2 \mu_{1}\right), & b \in B_{9} \\
0.5\left(N-2-2 \mu_{2}+2 \mu_{3}\right), & b \in B_{10}\end{cases}
\end{aligned}
$$

$$
\left|T_{3}\right|= \begin{cases}0, & b \in B_{2} \\ N-1, & b \in B_{1} \cup B_{3}\end{cases}
$$

and

$$
\left|T_{4}\right|=0.5\left(|U|-\left|T_{1}\right|-\left|T_{2}\right|\right)-\left|T_{3}\right|
$$

with $\mu_{1}=\bmod (0.5 N, 2), \mu_{2}=\bmod (b, 2), \mu_{3}=\bmod (b+$ $0.5 N, 2), B_{4}=\{0\}, B_{5}=\{1\}, B_{6}=\{2, \ldots, 0.5 N-1\}$, $B_{7}=\{0.5 N\}, B_{8}=\{0.5 N+1\}, B_{9}=\{0.5 N+2\}$, and $B_{10}=\{0.5 N+3, \ldots, N\} . \operatorname{By} \bmod \left(\lambda_{1}, \lambda_{2}\right)$, we denote the value of $\lambda_{1}$ modulo $\lambda_{2}$.

If $N=4 \cdot \nu, \nu \in \mathbb{N},\left|T_{2}\right|$ simplifies to

$$
\left|T_{2}\right|= \begin{cases}0.5 N, & b \in B_{2} \\ 0.5 N-1, & b \in B_{1} \cup B_{3}\end{cases}
$$

We note that, in the calculation of $\left|T_{2}\right|$, we have used the expansion (assume $x<1$ )

$$
\begin{aligned}
\frac{1}{1-x^{2}} \cdot \frac{1}{1-x} & =\sum_{n=0}^{\infty}\lfloor 0.5 n+1\rfloor x^{n} \\
& =\sum_{n=0}^{\infty}(0.5 n-0.5 \bmod (n, 2)+1) x^{n}
\end{aligned}
$$

with $\lfloor\cdot\rfloor$ denoting the floor function.

\section{APPENDIX II}

Sets Sizes in the CASE of AN AdJACENT CHANNEL OF DIFFERENT AMPLITUDE

In the case of an OFDM channel along with an adjacent channel of different amplitude, we have evaluated the sizes of the various sets. The sizes of sets $U, T_{4_{0}}, T_{4_{1}}, T_{4_{2}}, T_{4_{3}}$, and $T_{4_{4}}$ are given in (61) and (71)-(74), while the sizes of all other sets are given as follows:

$$
\begin{aligned}
& \left|T_{10}\right|= \begin{cases}0, & b \in B_{2} \\
1, & b \in B_{1} \cup B_{3}\end{cases} \\
& \left|T_{11}\right|=\left|T_{12}\right|=\left|T_{13}\right|=0 \\
& \left|T_{20}\right|= \begin{cases}0.5 N, \quad b \in B_{2} \\
0.5 N-1, \quad b \in B_{1} \cup B_{3}\end{cases} \\
& \left|T_{21}\right|= \begin{cases}0.5\left(N-g-b+\mu_{4}\right), & b \in B_{11} \\
0.5\left(N-g-b-\mu_{4}\right)+1, & b \in B_{12} \\
0, & b \in B_{13}\end{cases} \\
& \left|T_{22}\right|= \begin{cases}0, & b \in B_{14} \\
0.5\left(b-g+\mu_{4}\right)+1, & b \in B_{15} \\
0.25 N, & b \in B_{16} \\
0.5\left(b-g+2 \mu_{4}\right)+1, & b \in B_{17}\end{cases} \\
& \left|T_{23}\right|=0 \\
& \left|T_{30}\right|= \begin{cases}0, & b \in B_{2} \\
N-1, & b \in B_{1} \cup B_{3}\end{cases}
\end{aligned}
$$

$$
|U|= \begin{cases}1-7.5 b-2.5 b^{2}+2 b N+2 N^{2}+b g-N g+0.5 g^{2}+1.5 g, & 0 \leq b<g \\ 4-3 b-b^{2}+2 b N+2 N^{2}-2 b g-N g+2 g^{2}-3 g, & g \leq b<0.5 N-g+2 \\ 7-6 b-b^{2}+2 b N+2 N^{2}-2 b g-N g+2 g^{2}-6 g+1.5 N, & 0.5 N-g+2 \leq b<0.5 N \\ -6 b-b^{2}+2 b N+2 N^{2}+6-2 b g-N g+2 g^{2}-6 g+1.5 N, & b=0.5 N \\ 7+12 b-b^{2}+2 b N+2 N^{2}-7.5 N-2 b g-N g+2 g^{2}-6 g, & 0.5 N<b<0.5 N+g \\ -2+3 g+3 b+2 b N-b^{2}-2 b g+2 g^{2}-N g-3 N+2 N^{2}, & 0.5 N+g \leq b<N+3-g \\ -3+4.5 g+4.5 b+3 b N-3 b g-4.5 N-1.5 b^{2}+1.5 N^{2}+1.5 g^{2}, & N+3-g \leq b \leq N\end{cases}
$$




$$
\begin{aligned}
& \left|T_{31}\right|=\left|T_{33}\right|=0 \\
& \left|T_{32}\right|=N
\end{aligned}
$$

where $\mu_{4}=\bmod (b+g, 2), B_{11}=\{0, \ldots, 0.5 N+1-g\}$, $B_{12}=\{0.5 N+2-g, \ldots, N+2-g\}, B_{13}=\{N+3-$ $g, \ldots, N\}, B_{14}=\{0, \ldots, g-1\}, B_{15}=\{g, \ldots, 0.5 N+g-1\}$, $B_{16}=\{0.5 N+g\}$, and $B_{17}=\{0.5 N+g+1, \ldots, N\}$. The number of carriers $N$ is assumed to satisfy $N=4 \cdot \nu$ and $\nu \in \mathbb{N}$.

\section{APPENDIX III}

SET SiZES IN THE CASE OF AN ARBITRARY

NUMBER OF ADJACENT CHANNELS

We have evaluated the sizes of the various sets in the case of an arbitrary number of adjacent OFDM channels of the same amplitude as follows:

$$
|U|= \begin{cases}\left|U_{1}\right|, & 0 \leq b<g \\ \left|U_{2}\right|, & g \leq b \leq N\end{cases}
$$

where

$$
\begin{aligned}
\left|U_{1}\right|= & u_{b+N+r(N+g)}-3 u_{b-1+r(N+g)} \\
& +3 u_{b-N-2+r(N+g)}-u_{b-2 N-3+r(N+g)} \\
\left|U_{2}\right|= & u_{b+N+r(N+g)}-3 u_{b-1+r(N+g)}-3 u_{b-g} \\
& +3 u_{b-N-2+r(N+g)}-u_{b-2 N-3+r(N+g)} \\
\left|T_{1}\right|= & 1 \\
\left|T_{2}\right|= & \begin{cases}\left|S_{1}\right|, \quad 0 \leq b<g \\
\left|S_{2}\right|, \quad g \leq b \leq N\end{cases} \\
\left|T_{3}\right|= & N+r(N+1) \\
\left|T_{4}\right|= & 0.5\left(|U|-\left|T_{1}\right|-\left|T_{2}\right|\right)-\left|T_{3}\right|
\end{aligned}
$$

where

$$
\begin{aligned}
\left|S_{1}\right|= & s_{b+N+r(N+g)}-s_{b+r(N+g)-1} \\
& +s_{b-2 N+r(N+g)}-s_{b-N+r(N+g)} \\
\left|S_{2}\right|= & s_{b+N+r(N+g)}-s_{b+r(N+g)-1}-s_{b-g} \\
& +s_{b-2 N+r(N+g)}-s_{b-N+r(N+g)}
\end{aligned}
$$

and

$$
s_{n} \doteq \sum_{l=0}^{t} p[n-(t-l)(N+g)] \cdot p[t-l] .
$$

We define $p[n]=\lfloor(n / 2)+1\rfloor$ while $t$ is defined in (47).

\section{APPENDIX IV}

\section{CROSS-CORRELATION OF IMD SETS}

In order to calculate the intermodulation noise in sets $T_{2}$ and $T_{4}$, we need to evaluate the cross-correlations of the following.

(i) Any two distinct terms in set $T_{2}$.

(ii) Any two distinct terms in set $T_{4}$.

(iii) Any two terms in sets $T_{2}$ and $T_{4}$

Lemma 1: We assume that the RVs $\theta_{i}$ satisfy the following.

(a) They are independent and identically distributed.

(b) Their probability density function is symmetrical about $\theta_{i}=0$ and $\theta_{i}=\pi / 2$.

We construct the random processes $e^{j \theta_{\xi}}$, where

$$
\theta_{\xi}=\theta_{\xi_{+}}-\theta_{\xi_{-}}
$$

and with $n$ odd, we define

$$
\begin{aligned}
\theta_{\xi_{+}} & =\sum_{\zeta=1}^{\frac{n+1}{2}} \theta_{i}(\zeta) \\
\theta_{\xi_{-}} & =\sum_{\zeta=1}^{\frac{n-1}{2}} \theta_{i}(\zeta) .
\end{aligned}
$$

The thus generated random processes $e^{j \theta_{\xi}}$ are zero mean.

\section{Proof of Lemma 1:}

Remark 1: Due to condition (b), the $\mathrm{RVs} e^{j \theta_{i}}$ are zero mean.

1) In the case of $n=1, \theta_{\xi_{-}}=0$ and $\theta_{\xi_{+}}=\theta_{i}$ and Remark 1 ensures that $\left\langle e^{j \theta_{\xi}}\right\rangle=0$.

$$
\begin{aligned}
& \left|T_{4_{0}}\right|= \begin{cases}1.5-1.5 b-0.5 b^{2}+0.5 b N+0.25 N^{2}-1.25 N, & 0 \leq b<0.5 N \\
0.375 N^{2}-N, & b=0.5 N \\
1.5+1.5 b-0.5 b^{2}+0.5 b N+0.25 N^{2}-2.75 N, \quad 0.5<b \leq N\end{cases} \\
& \left|T_{4_{1}}\right|= \begin{cases}-1+0.5(b g-b N-N g)+0.25\left(b^{2}+N^{2}+g^{2}-N-\mu_{4}\right)+g+b, & 0 \leq b<g \\
0.5(5 b-b N-b g-N g-g)+0.25\left(N^{2}-N+3 b^{2}+3 g^{2}-\mu_{4}\right), & g \leq b<0.5 N-g+2 \\
1+0.5(N-b N-b g-N g)+0.25\left(3 b^{2}+N^{2}+3 g^{2}+\mu_{4}\right)+b-2 g, & 0.5 N-g+2 \leq b<0.5 N+g \\
-2-0.5(b N+b g+N g)+0.25\left(N^{2}+3 g^{2}+3 b^{2}+\mu_{4}\right)-2 b+2 N+g, & 0.5 N+g \leq b<N+3-g \\
-2+0.5\left(3 g-3 b+3 N+b^{2}+g^{2}\right)-b g, & N+3-g \leq b \leq N\end{cases} \\
& \left|T_{4_{2}}\right|= \begin{cases}1-b^{2}+b N-3 b+0.5 N^{2}-N, & 0 \leq b<g \\
0.5-b^{2}+b N-3.25 b+0.5 N^{2}+0.25 g-N-0.25 \mu_{4}, & g \leq b<0.5 N \\
0.5-b^{2}+b N+2.75 b+0.5 N^{2}-4 N+0.25 g-0.25 \mu_{4}, & 0.5 N \leq b \leq 0.5 N+g \\
1-b^{2}+b N+3 b+0.5 N^{2}-4.125 N, & b=0.5 N+g \\
0.5-b^{2}+b N+2.75 b+0.5 N^{2}-4 N+0.25 g-0.5 \mu_{4}, & 0.5 N+g+1 \leq b \leq N\end{cases}
\end{aligned}
$$

$$
\left|T_{4_{3}}\right|= \begin{cases}0 & 0 \leq b<g \\ 0.5+0.25 b^{2}-0.5 b g+0.75 b+0.25 g^{2}-0.75 g & g \leq b<0.5 N+g \\ -1+0.25 b^{2}-0.5 b g-0.75 b+0.25 g^{2}+0.75 g+0.75 N, & 0.5 N+g \leq b \leq N\end{cases}
$$


2) For $n>1$, either $(n+1) / 2$ or $(n-1) / 2$ is odd so that either $\theta_{\xi_{+}}$or $\theta_{\xi_{-}}$will include a variable $\theta_{s}$ with unit multiplicity. Due to condition (a),

$$
\left\langle e^{j \theta_{\xi}}\right\rangle=\left\langle e^{j \theta_{\xi}-\theta_{s}} e^{j \theta_{s}}\right\rangle=\left\langle e^{j \theta_{\xi}-\theta_{s}}\right\rangle\left\langle e^{j \theta_{s}}\right\rangle=0
$$

The criteria of Lemma 1 are satisfied in the case of $M$-QAM and DQPSK modulations. As a result, in both $T_{2}$ and $T_{4}$, the IMD products are zero mean. Furthermore, for any distinct pair of IMD products, the relevant angles $\theta_{\xi}$ are uncorrelated. Therefore, the cross-correlations in cases (i)-(iii) are estimated as null.

\section{ACKNOWLEDGMENT}

The authors would like to thank the anonymous reviewers for their valuable comments.

\section{REFERENCES}

[1] W. Zou and Y. Wu, "COFDM: An overview," IEEE Trans. Broadcast., vol. 41, no. 1, pp. 1-8, Mar. 1995.

[2] T. Keller and L. Hanzo, "Adaptive multicarrier modulation: A convenient framework for time-frequency processing in wireless communications," Proc. IEEE, vol. 88, no. 5, pp. 611-640, May 2000.

[3] X. Li and L. J. Cimini, "Effects of clipping and filtering on the performance of OFDM," IEEE Commun. Lett., vol. 2, no. 5, pp. 131-133, May 1998.

[4] S. Merchan, A. G. Armada, and J. Garcia, "OFDM performance in amplifier nonlinearity," IEEE Trans. Broadcast., vol. 44, no. 1, pp. 106-114, Mar. 1998.

[5] O. Shimbo, "Effects of intermodulation, AM-PM conversion, and additive noise in multicarrier TWT systems," Proc. IEEE, vol. 59, no. 2 , pp. 230-238, Feb. 1971

[6] D. Dardari, V. Tralli, and A. Vaccari, "A theoretical characterization of nonlinear distortion effects in OFDM systems," IEEE Trans. Commun., vol. 48 , no. 10 , pp. $1755-1764$, Oct. 2000.

[7] G. Santella and F. Mazzenga, "A hybrid analytical-simulation procedure for performance evaluation in $M$-QAM-OFDM schemes in presence of nonlinear distortions," IEEE Trans. Veh. Technol., vol. 47, no. 1, pp. 142-151, Feb. 1998.

[8] P. Banelli, G. Baruffa, and S. Cacopardi, "Effects of HPA non linearity on frequency multiplexed OFDM signals," IEEE Trans. Broadcast., vol. 47, no. 2, pp. 123-136, Jun. 2001.

[9] A. M. Saleh, "Frequency independent and frequency dependent nonlinear models of TWT amplifiers," IEEE Trans. Commun., vol. COM-29, no. 11, pp. 1715-1720, Nov. 1981.

[10] S. A. Maas, "How to model intermodulation distortion," in IEEE MTT-S Int. Microw. Symp. Dig., 1991, pp. 149-151.

[11] Q. Shi, "OFDM in bandpass nonlinearity," IEEE Trans. Consum. Electron., vol. 42, no. 3, pp. 253-258, Aug. 1996.

[12] C. van den Bos, M. H. L. Ksuwenhoven, and W. A. Serdijn, "Effect of smooth nonlinear distortion on OFDM symbol error rate," IEEE Trans. Commun., vol. 49, no. 9, pp. 1510-1514, Sep. 2001.

[13] W. B. Davenport and W. L. Root, An Introduction to the Theory of Random Signals and Noise. New York: McGraw-Hill, 1958.

[14] N. M. Blachman, "The signal $x$ signal, noise $x$ noise, and signal $x$ noise output of a nonlinearity," IEEE Trans. Inf. Theory, vol. IT-14, no. 1, pp. 21-27, Jan. 1968.

[15] E. Costa and S. Pupolin, " $M$-QAM-OFDM performance in the presence of a nonlinear amplifier and phase noise," IEEE Trans. Commun., vol. 50, no. 3, pp. 462-472, Mar. 2002.
[16] R. J. Westcott, "Investigation of multiple FM/FDM carriers through a satellite TWT operating near to saturation," Proc. IEEE, vol. 114, no. 6, pp. 726-740, Jun. 1967.

[17] J. C. Pedro and N. B. de Carvalho, "On the use of multitone techniques for assessing RF component's intermodulation distortion," IEEE Trans. Microw. Theory Tech., vol. 47, no. 12, pp. 2393-2402, Dec. 1999.

[18] C. Liu, "The effect of nonlinearity on a QPSK-OFDM-QAM signal," IEEE Trans. Consum. Electron., vol. 43, no. 3, pp. 443-447, Aug. 1997.

[19] N. Boulejfen, A. Harguem, and F. A. Channouchi, "New closed-form expressions for the prediction of multitone intermodulation distortion in fifth-order nonlinear RF circuits/systems," IEEE Trans. Microw. Theory Tech., vol. 52, no. 1, pp. 121-132, Jan. 2004.

[20] R. Deutsch, Non-Linear Transformations of Random Processes. New York: Prentice-Hall, 1962.

[21] A. Heiskanen and T. Rahkonen, "Fifth-order multi-tone Volterra simulator with component-level output," in Proc. Int. Circuits Syst. Symp., May 2002, vol. 3, 2, pp. 591-594.

[22] C. Chien, Digital Radio on a Chip. Norwell, MA: Kluwer, 2001.

[23] C. Fager, J. C. Pedro, N. B. de Carvalho, H. Zirath, F. Fortes, and M. J. Rosario, "A comprehensive analysis of IMD behavior in RF CMOS power amplifiers," IEEE J. Solid-State Circuits, vol. 39, no. 1, pp 24-34, Jan. 2004.

[24] J. Bradley, Applied Combinatorics With Problem Solving. Reading, MA: Addison-Wesley, 1990.

[25] A. Tucker, Applied Combinatorics, 3rd ed. New York: Wiley, 1994.

[26] U. Rodhe, RF/Microwave Circuit Design for Wireless Applications. New York: Wiley, 2000.

[27] J. G. Proakis, Digital Communications, 4th ed. New York: McGrawHill, 2001.

[28] J. Stott, "The effects of phase noise in COFDM," EBU Tech. Rev.,, vol 276, pp. 1-19, Jul. 1998

[29] J. C. Pedro and N. B. de Carvalho, "Characterizing nonlinear RF circuits for their in-band distortion," IEEE Trans. Instrum. Meas., vol. 51 no. 3 , pp. 420-426, Jun. 2002.

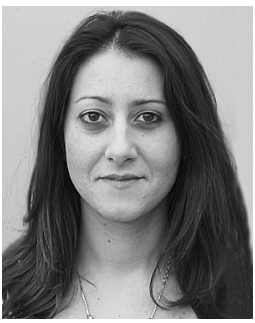

Arsenia Chorti received the M. Eng. degree in electrical engineering from the University of $\mathrm{Pa}$ tras, Patras, Greece, in 1998, the D.E.A. degree in electronics from the University Pierre et Marie Curie-Paris VI, Paris, France, in 2000, and the Ph.D. degree in communications and signal processing from Imperial College London, London, U.K., in 2005.

She is currently a Research Fellow with the Electronic Systems Design group, Electronics and Computer Science Department, University of Southampton, Southampton, U.K., where she is involved in the area of intelligent sensing. Her research interests include multicarrier communication systems-OFDM, stochastic signal processing, density estimation, novelty detection, and prediction/estimation algorithms.

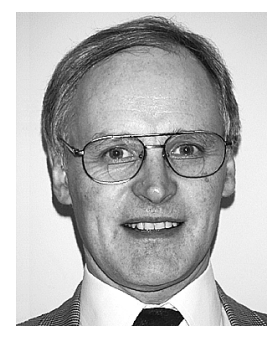

Mike Brookes (M'88) received the B.A. degree in mathematics from Cambridge University, Cambridge U.K., in 1972.

Upon graduation, he spent four years with the Massachusetts Institute of Technology (MIT), Cambridge, where he was involved with astronomical instrumentation and telescope control systems. Since 1979, he has been with the Electrical and Electronic Engineering Department, Imperial College London, London, U.K., where he is a Reader with the Communications and Signal Processing Research Group. His main areas of research are speech processing where he has been involved with speech production modeling, speaker recognition algorithms, and techniques for speech enhancement using both single microphones and microphone arrays. He currently applies techniques from speech processing to RADAR target identification and is also actively involved in computer vision research. 